\title{
Dissemination routes of the carbapenem resistance plasmid pOXA-48 in a
} hospital setting

Ricardo León-Sampedro1,2,a, Javier DelaFuente, ${ }^{1, a}$, Cristina Díaz-Agero,a, Thomas Crellen $^{4,5}$, Patrick Musicha ${ }^{4,5}$, Jerónimo Rodriguez-Beltran ${ }^{1,2}$, Carmen de la Vega ${ }^{1}$, Marta Hernández-García ${ }^{1,6}$, R-GNOSIS WP5 Study Group ${ }^{7}$, Nieves Lopez-Fresneña ${ }^{3}$, Patricia Ruiz-Garbajosa ${ }^{1,6}$, Rafael Canton ${ }^{1,6}$, Ben $S$ Cooper $^{4,5}$ and Alvaro San Millan ${ }^{1,2, *}$.

${ }^{1}$ Servicio de Microbiología. Hospital Universitario Ramón y Cajal and Instituto Ramón y Cajal de Investigación Sanitaria. Madrid, Spain.

${ }^{2}$ Centro de Investigación Biológica en Red. Epidemiología y Salud Pública, Instituto de Salud Carlos III. Madrid. Spain.

${ }^{3}$ Servicio de Medicina Preventiva y Salud Pública, Hospital Universitario Ramón y Cajal and Instituto Ramón y Cajal de Investigación Sanitaria. Madrid, Spain.

${ }^{4}$ Mahidol-Oxford Tropical Medicine Research Unit, Faculty of Tropical Medicine, Mahidol University, Bangkok, Thailand.

${ }^{5}$ Centre for Tropical Medicine \& Global Health, Nuffield Department of Medicine, University of Oxford, Oxford, United Kingdom.

${ }^{6}$ Red Española de Investigación en Patología Infecciosa. Instituto de Salud Carlos III. Madrid. Spain.

${ }^{7}$ R-GNOSIS WP5 Study Group (in alphabetical order, excluding members present in the authors list, CDA, NLF, PRG, RC and BSC): Michael Behnke, Hetty Blok, Marc Bonten, Christian Brun-Buisson, Carolina Fankhauser, Petra Gastmeier, Jennifer Golembus, Herman Goossens, Sonja Hansen, Stephan Harbarth, Benedikt Huttner, Fieke Kloosterman, Axel Kola, Andrea Kuperberg, Christine Lammens, Frank Leus, Friederike Maechler, Surbhi Malhotra, J.J. Schotsman, Frank Schwab, Carlos Triay.

${ }^{a}$ These authors contributed equally to this study.

* Correspondence: alvsanmillan@gmail.com ORCID: 0000-0001-8544-0387 


\section{Introductory paragraph}

Infections caused by carbapenemase-producing enterobacteria (CPE) are a major concern in clinical settings worldwide. Two fundamentally different processes shape the epidemiology of CPE in hospitals: the dissemination of CPE clones from patient to patient (between-patient transfer), and the transfer of carbapenemase-encoding plasmids between enterobacteria in the gut microbiota of individual patients (withinpatient transfer). The relative contribution of each process to the overall dissemination of carbapenem resistance in hospitals remains poorly understood. Here, we used mechanistic models combining epidemiological data from more than 9,000 patients with whole genome sequence information from 250 enterobacteria clones to characterise the dissemination routes of the carbapenemase-encoding plasmid pOXA48 in a hospital setting over a two-year period. Our results revealed frequent betweenpatient transmission of high-risk pOXA-48-carrying clones, mostly of Klebsiella pneumoniae and sporadically Escherichia coli. The results also identified pOXA-48 dissemination hotspots within the hospital, such as specific wards and individual rooms within wards. Using high-resolution plasmid sequence analysis, we uncovered the pervasive within-patient transfer of pOXA-48, suggesting that horizontal plasmid transfer occurs in the gut of virtually every colonised patient. The complex and multifaceted epidemiological scenario exposed by this study provides new insights for the development of intervention strategies to control the in-hospital spread of CPE.

\section{Introduction}

Antibiotic resistance is one of the most concerning health challenges facing modern societies $^{1}$. Antibiotic resistance is of particular concern in clinical settings, where resistant pathogens significantly increase the mortality rates of critically ill patients and the costs associated with infection management and control ${ }^{1,2}$. The spread of antibiotic resistance genes between bacteria commonly associated with nosocomial infections is mainly driven by the horizontal transfer of conjugative plasmids ${ }^{3,4}$. However, the 
frequency with which this occurs in the clinical settings and its importance for the dissemination of resistance at a local level remain poorly defined.

One of the most clinically relevant groups of nosocomial pathogens are enterobacteria that produce carbapenemases (ß-lactamase enzymes able to degrade carbapenem antibiotics). Among carbapenemase-producing enterobacteria (CPE), clones of Klebsiella pneumoniae and Escherichia coli carrying plasmid-encoded carbapenemases pose the highest clinical threat ${ }^{5}$. Despite their clinical relevance, major gaps remain in our understanding of the epidemiology of CPE and of carbapenemase-encoding plasmids. Previous work has highlighted the importance of in-hospital CPE transmission from patient to patient ${ }^{6,7}$ (between-patient transfer). However, the dissemination and evolution of CPE in hospitals present an additional layer of complexity: the transfer of carbapenemase-encoding plasmids between enterobacteria clones in the gut microbiota of individual patients (within-patient transfer $)^{8,9}$. Understanding the relative importance of between-patient and withinpatient transfer is of central importance for understanding the epidemiology of CPE and informing intervention strategies to control the spread of carbapenem resistance in clinical settings.

One of the most frequent carbapenemases in enterobacteria is OXA-48 ${ }^{10}$. OXA-48 was first described in a K. pneumoniae strain isolated in Turkey in $2001^{11}$ and is now distributed worldwide, with particularly high prevalence in North Africa, Middle Eastern countries, and Europe ${ }^{10}$. The blaoxA-48 gene is usually encoded in an IncL, broad-hostrange conjugative plasmid called pOXA- $48^{8}$ (Figure S1). This plasmid is frequently associated with K. pneumoniae high-risk clones ${ }^{12}$, such as the sequence types 11 (ST11), ST15, ST101, and ST405,13-15, which are able to readily spread between hospitalized patients producing outbreaks of infections ${ }^{16,17}$. Previous epidemiological studies strongly suggested the possibility of within-patient pOXA-48 transfer $^{17-21}$, 
indicating that pOXA-48 would be an ideal study system to investigate the nosocomial dissemination of carbapenem resistance.

In the present study, we examined the between-patient and within-patient transfer dynamics of plasmid pOXA-48 in a tertiary hospital over a two-year period. For our analysis, we used a large and well-characterized collection of pOXA-48-carrying enterobacteria generated at the Hospital Universitario Ramon y Cajal in Madrid as part of the European project R-GNOSIS (Resistance of Gram-Negative Organisms: Studying Intervention Strategies) ${ }^{22,23}$. Using statistical models and combining epidemiological data from more than 9,000 patients with whole-genome sequence information from 250 enterobacteria clones, we aimed to define pOXA-48 transfer dynamics at an unprecedented resolution. Specifically, we aimed to determine the relative contribution of between-patient and within-patient plasmid transfer in the epidemiology of pOXA-48, and to use these data to inform improved intervention strategies to control the spread of carbapenem resistance in hospitals.

\section{Results}

Patients colonised by pOXA-48-carrying enterobacteria in the hospital

During the R-GNOSIS project, hospitalised patients were periodically sampled to detect the presence of enterobacteria producing extended spectrum ß-lactamases (ESBL) and carbapenemases in their gut microbiota (see methods). The study enrolled all patients admitted to two medical wards (gastroenterology and pneumology) and two surgical wards (neurosurgery and urology) in the hospital. The full details of the RGNOSIS study in our hospital, including the study population and CPE characterization, have been previously reported by Hernandez-Garcia et al ${ }^{18,23}$. Briefly, from March 2014 to March 2016, 28,089 rectal swabs were collected from 9,275 patients, and 171 pOXA-48-carrying enterobacteria strains were isolated and characterised from 105 patients (Figure 1, Table S1). The proportion of patients who 
were found to be colonized with pOXA-48-carrying enterobacteria on at least one occasion during their hospital admission was $0.5 \%$ in urology $(18 / 3,483), 1.3 \%$ in gastroenterology $(33 / 2,591)$, and $1.5 \%$ both in neurosurgery $(16 / 1,068)$ and pneumology $(38 / 2,559)$, with the medical wards accounting for $68 \%$ of colonised patients $(71 / 105$, Figure $1 A-C)$.

In line with previous reports ${ }^{10}, K$. pneumoniae was the most frequent pOXA-48carrying species $(n=108)$. However, pOXA-48 was detected in an additional 7 enterobacterial species, with $E$. coli being the second most frequent carrier $(n=45$, Figure 1D, Table S1). In several pOXA-48 carrying patients (18/105), there was cocolonisation of the gut microbiota with more than one species carrying the plasmid, suggestive of within-patient plasmid transfer events (Figure 1C).

A
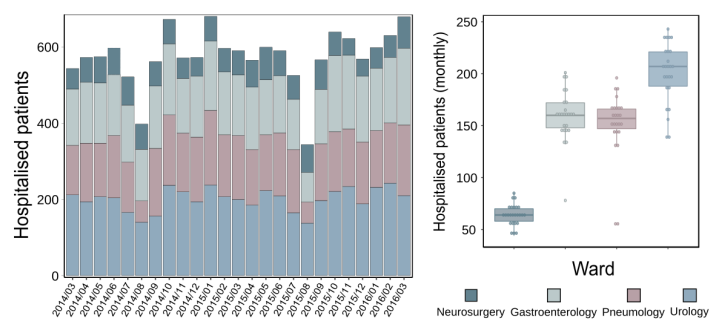

C

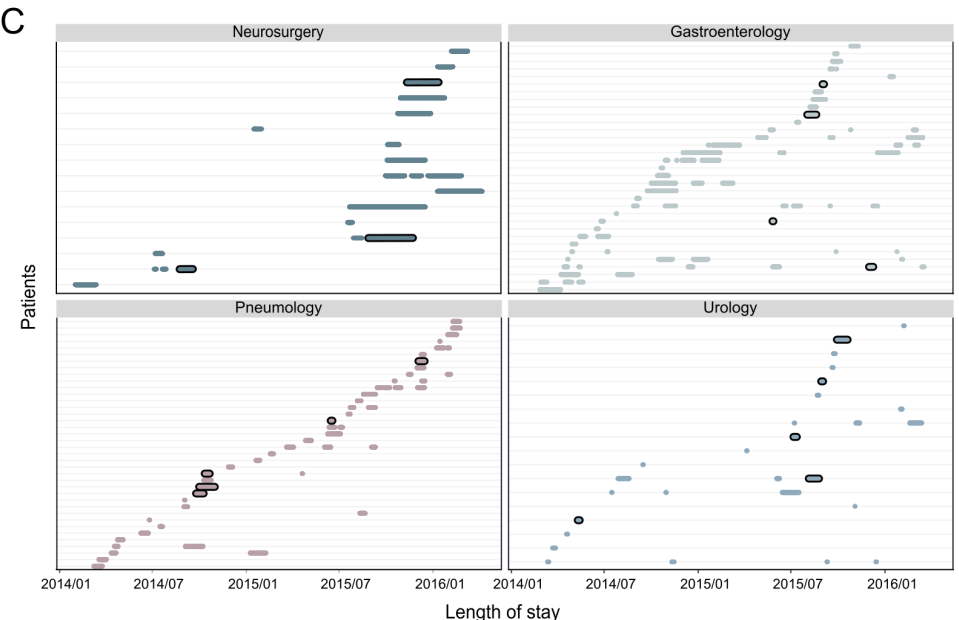

B
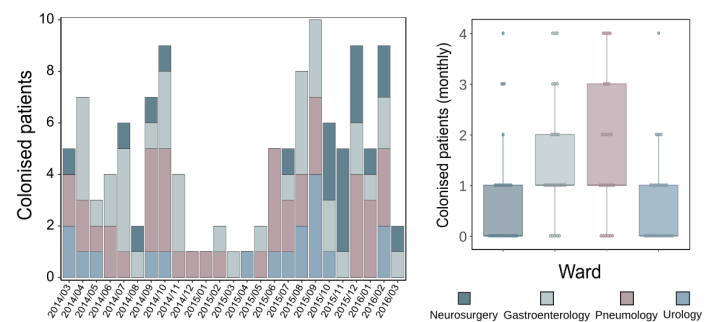

D

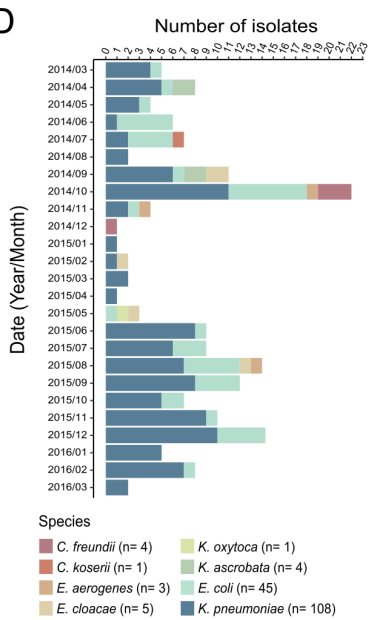

$\overline{\text { Figure 1. Study population, colonised patients, and pOXA-48-carrying enterobacteria. }}$ (A) Patients sampled during the R-GNOSIS study. The left panel shows the number of hospitalised patients in each ward over the 25-month study period. The right panel shows the distribution of hospitalised patients per ward by month as a boxplot. 
Horizontal lines inside boxes indicate median values, the upper and lower hinges correspond to the 25th and 75th percentiles, and whiskers extend to observations within 1.5 times the interquartile range. (B) Patients colonised by a pOXA-48-carrying enterobacteria during the study. The left panel represents the number of colonised patients in each ward over the 25-month study period. The right panel shows the distribution of colonised patients per ward by month as a boxplot. (C) Distribution of patients colonised by pOXA-48-carrying enterobacteria in the four wards under study over the 25-month study period. Each row represents a patient, and the colour segments represent the length of hospital stay (from admission to discharge). Black outlining of colour segments indicates patient co-colonisation with more than one pOXA-48-carrying species. (D) Enterobacteria isolates carrying pOXA-48 recovered from the patients during the 25 months of the study. The species of the pOXA-48carrying isolates are colour-coded as indicated in the legend.

Using epidemiological data to analyse pOXA-48 transfer dynamics.

To investigate how pOXA-48 spread in the hospital, we analysed the epidemiological data using a previously described model which enabled us to estimate the daily probability of a patient acquiring pOXA-48-carrying enterobacteria and quantify the effect of covariates on this probability (see methods and Supplementary Table 2) ${ }^{24}$. We performed this analysis independently for the two species with a large number of isolates, K. pneumoniae and E. coli, and we included two covariates in the model. The first covariate was the number of other patients colonized by pOXA48-carrying enterobacteria in the ward on the same day, which we expect to be positively associated with the daily risk of acquisition if between-patient bacterial transfer is important. The second covariate was known pre-existing intestinal colonisation of the patient by a pOXA48-carrying enterobacteria of a different species $(K$. pneumoniae or E. coli). If within-patient plasmid transfer is important (from K. pneumoniae to E. coli and vice versa, Figure 2), then we would expect this to also be positively associated 
with the daily risk of a patient acquiring pOXA-48-carrying enterobacteria. We considered different transmission models including and excluding these covariates and performed model comparisons using the widely applicable information criterion (WAIC, Supplementary Table 2). The model that best fitted our data was the one including both covariates and permitting the transmission parameter $\beta$ to vary by ward (see Supplementary Table 3 for daily probability values and methods for details).

The baseline daily probabilities for becoming colonised with pOXA-48-carrying $K$. pneumoniae or E. coli were $0.1 \%$ (95\% credible interval [Crl] $0.08 \%, 0.12 \%)$ and $0.04 \%$ (95\% Crl, 0.02\%, 0.05\%), respectively (Supplementary Table 3). Results showed that the probability of acquisition of a pOXA-48-carrying $K$. pneumoniae was higher if other patients were already colonised with a pOXA-48-carrying clone in the wards of neurosurgery (Odds ratio [OR] 6.7, 95\% Crl 2.5, 11.7) and pneumology (OR 2.7, 95\% Crl 1.2, 4.6). In the wards of gastroenterology (OR 1.7, 95\% Crl 0.4, 4.1) and urology (OR $0.6,95 \% \mathrm{Crl} 0.01,4.4$ ) there were no clear effects. In contrast, the presence of other patients colonised by pOXA-48-carrying clones was not associated with the probability of acquiring a pOXA-48-carrying $E$. coli in the wards of neurosurgery (OR $0.23,95 \% \mathrm{Crl} 0.001,2.0$ ) or urology (OR $0.4,95 \% \mathrm{Crl} 0.002,2.7)$, and there was only weak evidence for a positive association in the wards of gastroenterology (OR 1.9, 95\% Crl 0.4, 4.7) and pneumology (OR 2.6, 95\% Crl 0.9, 4.5) (Figure 2A). This result suggested that $K$. pneumoniae is more important for between-patient transfer than $E$. coli.

The model also showed that prior colonisation with a pOXA-48-carrying clone of a different species was associated with a dramatic increase in the probability of acquiring a second pOXA48-carrying species (Figure $2 \mathrm{~A}, \mathrm{C}$ ). This risk was high both when a patient was first colonised by K. pneumoniae (OR 23.3, 95\% Crl 8.3, 53.4) and when initially colonized with E. coli (OR 15.8, 95\% Crl 3.8, 42.7). This result underlines the potential importance of within-patient plasmid transfer in the disemination of pOXA-48, 
a role supported by the high frequency of co-colonised patients (Figure $2 \mathrm{C}$ ). However, other explantions may be responsible for this observation, such as independent colonisation events of predisposed patients by different pOXA-48-carrying clones.

182
A

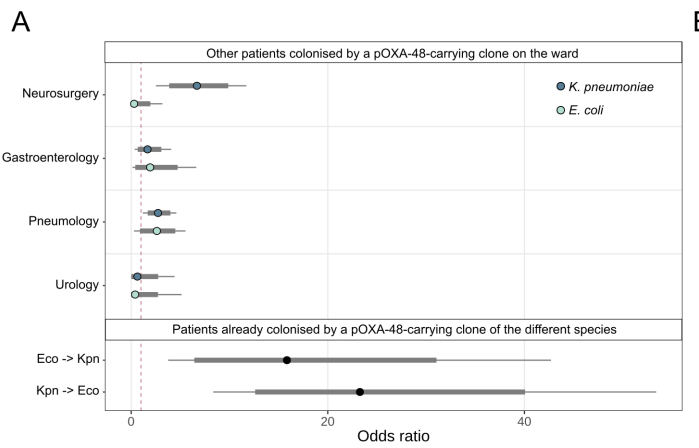

B

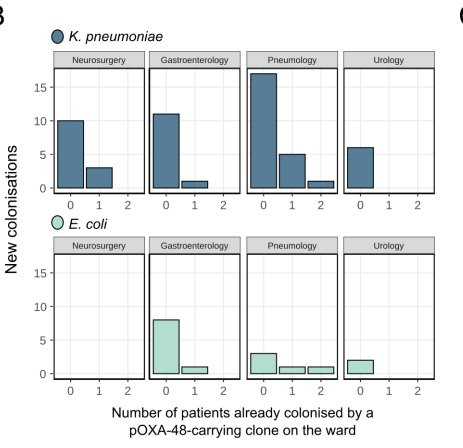

C

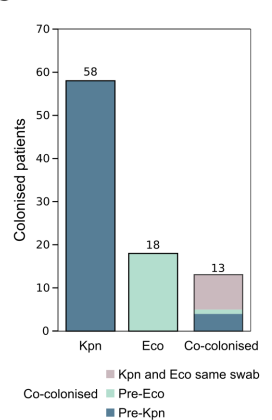

Figure 2. Acquisition of pOXA-48-carrying enterobacteria by hospitalised patients. (A) Posterior distribution of odds ratio for the daily risk of colonisation with a pOXA-48carrying K. pneumoniae or E. coli. Two covariates were included. The first is the presence of other patients colonised by a pOXA-48-carrying clone on the ward, (upper part, stratified by ward). If between-patient transfer of the plasmid is important, we expect to see a positive association (odds ratio $>1$ ) with the daily probability of acquiring a pOXA-48 clone. Second, pre-existing colonisation with a pOXA-48 clone of a different species (lower part). This covariate measures how being previously colonised by a pOXA-48-carrying $E$. coli is associated with the daily probability of becoming colonized with a pOXA-48-carrying K. pneumoniae clone (Eco -> Kpn) and vice versa (Kpn -> Eco). We expect to see a positive association if within-patient transfer of pOXA-48 between different bacterial clones is important. Points represent posterior medians; thick grey lines represent the $80 \% \mathrm{Crl}$ and thinner black lines represent the $95 \% \mathrm{Crl}$. (B) Number of previously uncolonised patients becoming colonised by a pOXA-48-carrying $K$. pneumoniae (top row) or E. coli (bottom row) as a function of the number of patients on the ward already colonised by a pOXA-48carrying clone. (C) Number of R-GNOSIS study patients colonised by pOXA-48carrying K. pneumoniae (Kpn) or E. coli (Eco) clones or both (co-colonised). For cocolonised patients, the colour code indicates whether K. pneumoniae or E. coli were 
isolated first or whether both species were simultaneously isolated from the same swab.

\section{Genomic analysis of pOXA-48-carrying enterobacteria}

A key limitation of our epidemiological model is that it is based solely on species identification, which restricts the possibility of reconstructing the spread of specific clones and plasmids. To track within-patient and between-patient plasmid transfer at a higher level of resolution, we integrated genomic information by sequencing the genomes of the 171 pOXA-48-carrying isolates represented in Figure 1D. In line with previous studies ${ }^{22,25}$, the sequencing results revealed that a small subset of isolates initially identified as K. pneumoniae actually belonged to the species Klebsiella quasipneumoniae $(\mathrm{n}=2)$ and Klebsiella variicola $(\mathrm{n}=3)$ (Supplementary Figure 2).

We analysed the genetic relatedness of isolates belonging to $K$. pneumoniae and to E. coli separately by reconstructing the core genome phylogeny for each species (Figure 3). For K. pneumoniae $(n=103)$, most of the isolates belonged to a few highrisk sequence types: ST11 $(n=64)$, ST307 $(n=17)$, and ST15 $(n=9)$. In contrast, $E$. coli $(n=45)$ showed a more diverse population structure, with only one sequence type, ST10, comprising more than three isolates $(n=11)$.

We next considered the distribution of the different clonal groups (defined by the different STs) across colonised patients (Figure 3A,B). Most $K$. pneumoniae isolates belonged to STs present in more than one patient, whereas approximately half of $E$. coli isolates belonged to STs present in only one patient (Figure 3C). This result, together with the results of statistical analysis, suggested that a limited number of $K$. pneumoniae high-risk clones are responsible for most of the between-patient transfer events. However, we observed several cases of pOXA-48-carrying E. coli STs colonising different patients, suggesting that $E$. coli is also responsible for sporadic between-patient transmission events. 


\section{A Klebsiella pneumoniae}

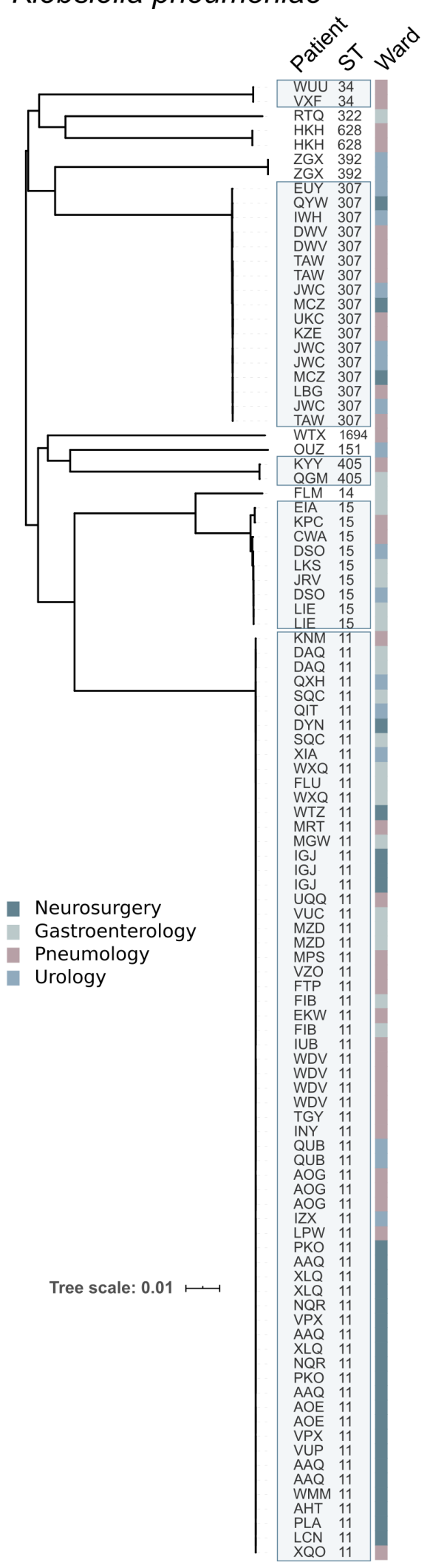

\section{B Escherichia coli}

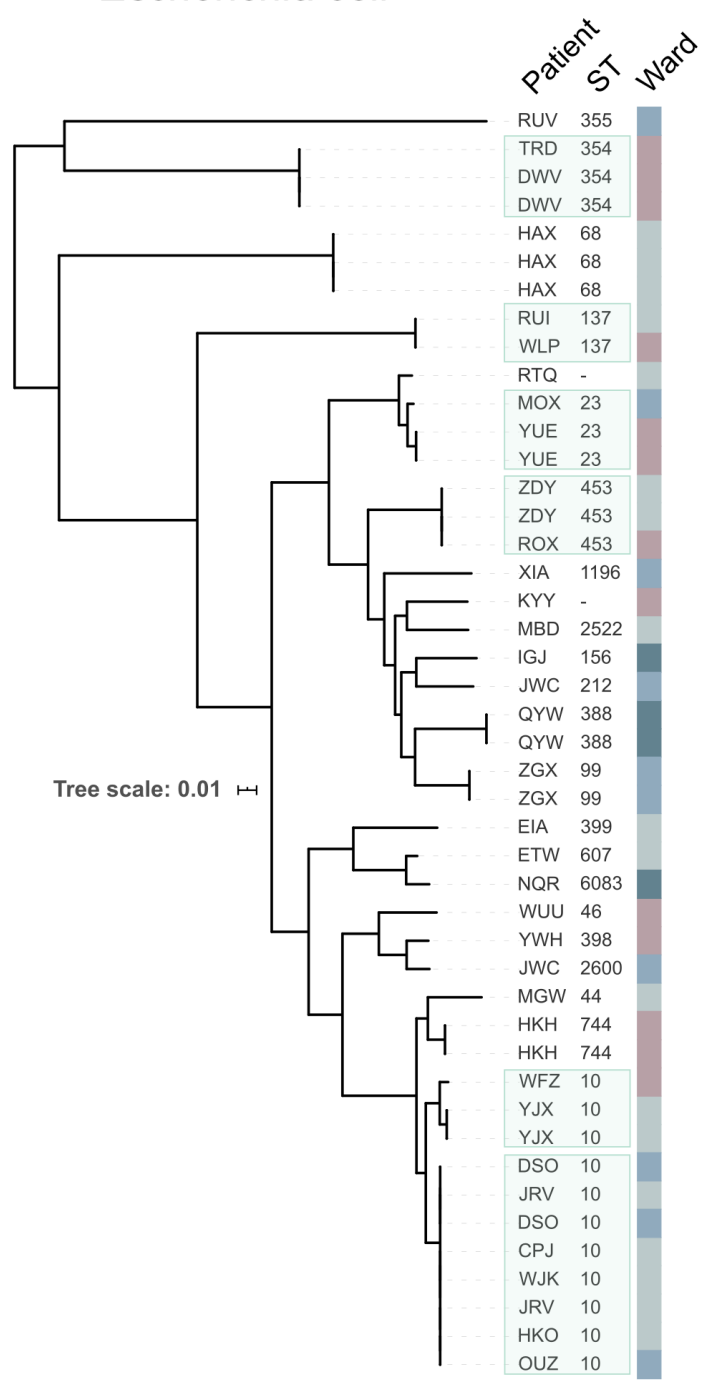

C

Isolates belonging to STs detected

in more than one patient

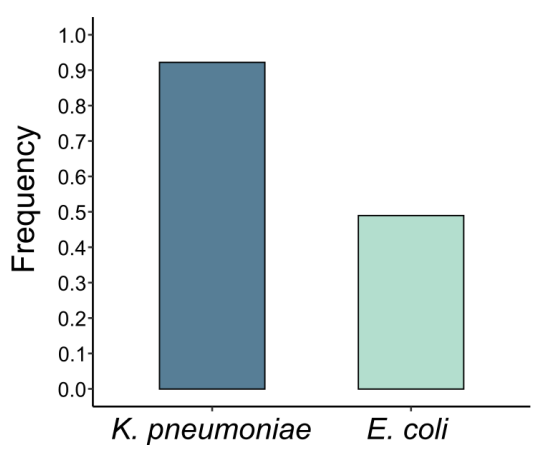


isolates carrying pOXA-48 and recovered during the R-GNOSIS study. Tree construction is based on polymorphisms in the core genome (scale: single nucleotide polymorphism [SNPs]/site). The columns to the right of the tree indicate patient code, isolate sequence type (ST), and the ward where the isolate was recovered (colour code in legend). Boxes with colour shading indicate recovery of isolates of the same sequence type (ST) from multiple patients in the hospital. (C) Frequency of pOXA-48carrying $K$. pneumoniae and E. coli isolates belonging to STs detected in multiple patients.

Reconstruction of between-patient transfer dynamics of pOXA-48-carrying clones.

To investigate the specific dissemination routes of pOXA-48-carrying clones, we integrated epidemiological and genomic data using SCOTTI ${ }^{26}$ (see methods). SCOTTI is a structured coalescent-based tool for reconstructing bacterial transmission, which accounts for bacterial diversity and evolution within hosts, non-sampled hosts, and multiple infections of the same host. We analysed the spread of the dominant $K$. pneumoniae and E. coli STs within and among the four wards under study (Figure 4, Supplementary Figures 3-10). As expected from the genomic data (Figure 3A), clones belonging to $K$. pneumoniae ST11 were responsible for most of the putative betweenpatient transmission events. The analysis attributed transmission events of pOXA-48carrying ST11 on every single ward and even between wards, with neurosurgery being the ward with the highest frequency and probability of ST11 transmission (Figure 4), 251 as suggested by the epidemiological model (Figure 2A). In light of these results, we 252 investigated K. pneumoniae ST11/pOXA-48 epidemiology in the neurosurgery ward in 253 more detail by looking at the spatiotemporal distribution of colonised patients 254 (Supplementary Figure 11). The neurosurgery ward includes 11 rooms with 20 beds 255 (9 double rooms and 2 single rooms). Of the 16 colonized patients, 6 had overlapping stays in the same room, suggesting that this room acted as a hotspot for $K$. pneumoniae ST11/pOXA-48 colonisation and transmission. 
SCOTTI also predicted transmission events mediated by three further pOXA-48carrying clones. Two transmission events were attributed to K. pneumoniae ST307 in the pneumology ward and three more to K. pneumoniae ST15: two in gastroenterology and another one between the gastroenterology and urology wards. In line with the genomic results (Figure 3B), SCOTTI also attributed two between-patient transfer events to $E$. coli ST10, one on the gastroenterology ward and another one between the gastroenterology and urology wards (Figure 4).

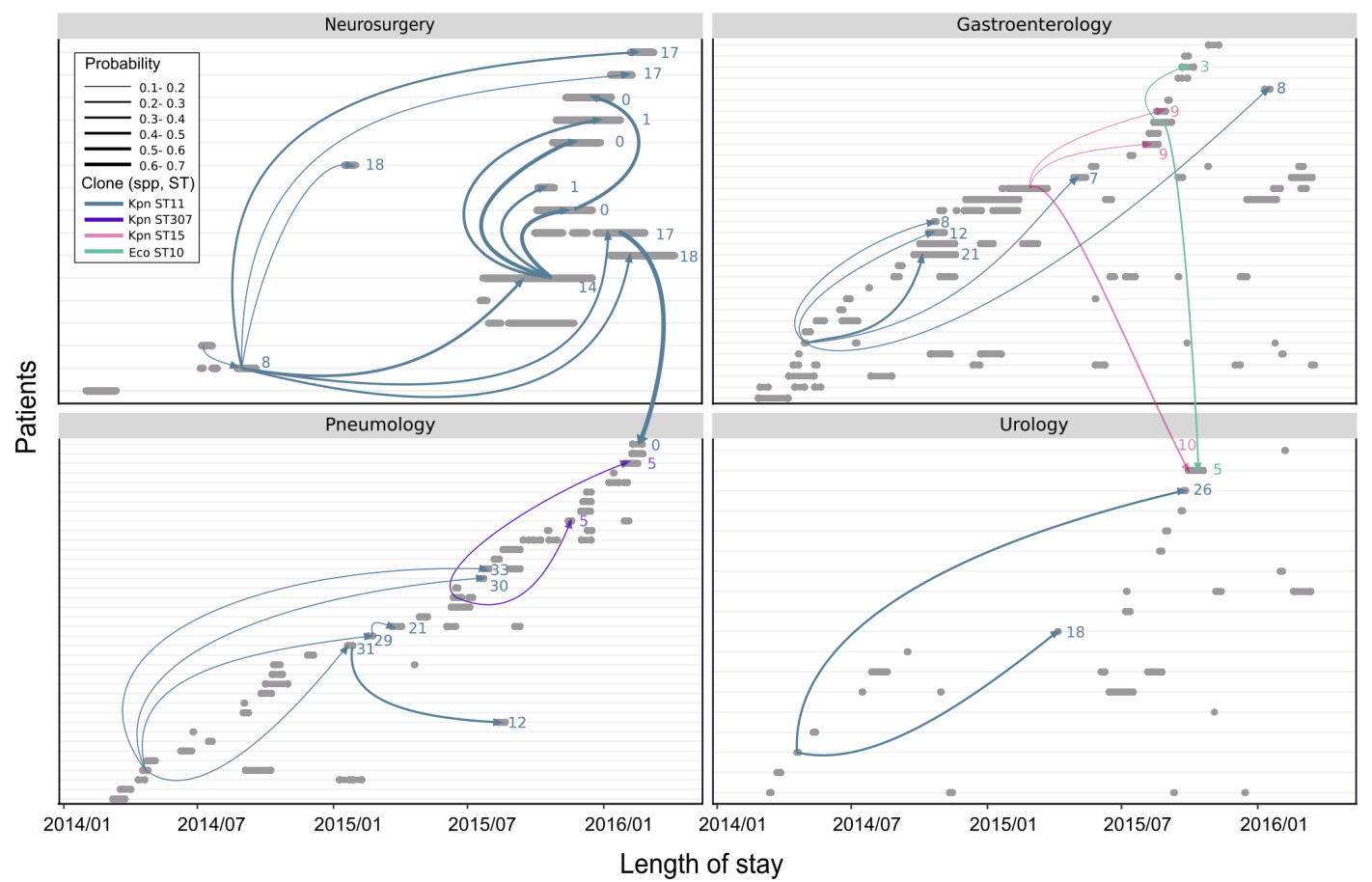

$\overline{\text { Figure 4. SCOTTI reconstruction of between-patient transfer of pOXA-48-carrying }}$ enterobacteria. The charts represent SCOTTI-attributed between-patient transfer events involving pOXA-48-carrying enterobacteria clones in the hospital, with individual panels representing the distribution of patients colonized by pOXA-48270 carrying enterobacteria on the different wards. Each row represents an individual 271 patient, and the grey segments represent the length of stay (from admission to 272 discharge). Coloured arrows represent transmission events predicted by SCOTTI. Line 273 colour indicates the clone responsible for the transmission event, and line thickness 274 represents the probability of the SCOTTI-attributed transmission, as indicated in the 
275 legend: Kpn, K. pneumonia; Eco, E. coli; ST, sequence type. Numbers to the right of 276 arrowheads indicate the number of SNPs differentiating the complete genomes of the 277 clone pair involved in the putative transmission event.

Genetic analysis of pOXA-48 confirms pervasive within-patient plasmid transfer

279 Our results suggest that the high frequency of patient colonisation by two plasmid280 carrying species could be due to within-patient pOXA-48 transfer (Figures 1 and 2). 281 However, although unlikely, another possibility is independent colonisation events involving different plasmid-carrying clones. To distinguish between these possibilities, we analysed the genetic sequence of plasmid pOXA-48 across all isolates with the aim of using specific genetic signatures in the plasmid to provide evidence for or against within-patient plasmid transfer. To increase the resolution of this analysis, we enriched the R-GNOSIS collection by recovering and sequencing the complete genomes of all the pOXA-48-carrying enterobacteria isolated from patients in our hospital since the plasmid was first reported in 2012 (Supplementary Table 1). In total, we determined and analysed the complete genomes of 250 strains, combining short-read and longread sequencing technologies (see methods and Supplementary Figure 12).

The results showed that pOXA-48 is highly conserved (Figure $5 A$ ). The core plasmid sequence spanned more than $60 \mathrm{~kb}$ ( $>90 \%$ of plasmid sequence) in 218 of the 250 strains (Supplementary Table 1). Analysis of the core genome among these 218 plasmids revealed an identical sequence in $80 \%$ of them. In the remaining $20 \%$, we detected a total 21 SNPs, with each plasmid presenting 1 or 2 SNPs compared with the most common variant (Figure 5A). This high degree of plasmid structural and sequence conservation and the strong link between pOXA-48 and the blaoxA-48 gene are important differences from previous analyses on the spread of plasmid-mediated carbapenemases such as $\mathrm{KPC}^{8,9,27}$.

Given the low plasmid-sequence variability, we could not track plasmid transmission 
transfer by using the rare plasmid variants carrying specific core-region SNPs as genetic fingerprints (Figure 5). We focused on instances where the same traceable plasmid variant was present in different bacterial clones (belonging to different species). We considered that isolation of different bacterial species carrying the same rare plasmid variant from the same patient would be a very strong indicator of withinpatient plasmid transfer. We found four examples in which the same rare plasmid variant was present in different bacterial species (Figure 5A). In all four, different species carrying the same plasmid variant were isolated from the same patient (Figure $5 B$ ). For example, plasmid variant 3 was detected in 6 bacterial isolates belonging to four clones (one K. pneumoniae, one E. coli and two C. freundii), and all of them were recovered from a single patient in the hospital (patient code YUE). Crucially, the chances of independent patient colonisation with the different bacterial clones carrying these rare plasmid variants are extremely low (variant $1,6.4 \times 10^{-4}$; variant $2,8.9 \times 10^{-4}$; variant $3,1.1 \times 10^{-8}$; variant $4,2.1 \times 10^{-5}$ ), confirming that these were within-patient plasmid transfer events.
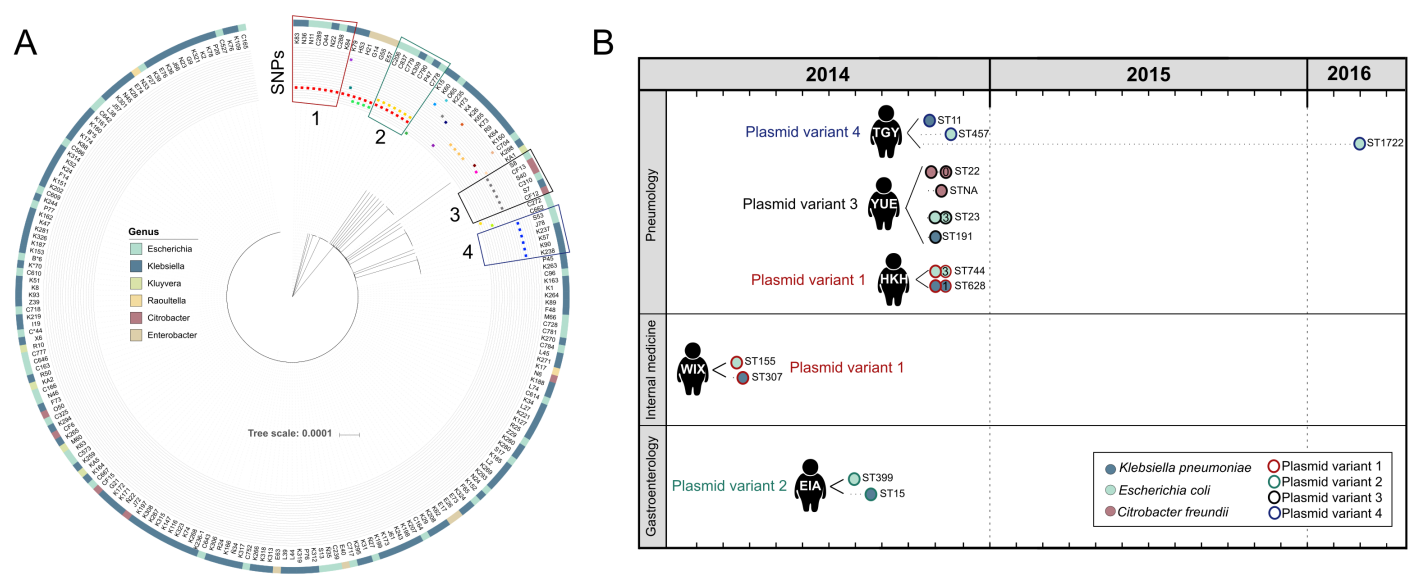

Figure 5. Pervasive within-patient pOXA-48 transfer. (A) Dendrogram constructed from the 21 polymorphisms present in the core region of plasmid pOXA-48. The outermost circle indicates plasmid-carrying clones genus according to the colour code in the legend, the second circle indicates the clone names, and the remaining circles indicate the presence of each plasmid SNP. Coloured boxes indicate the four plasmid variants 
carrying a 'rare' SNP present in clones of different species and used as genetic fingerprints. (B) Representation of patients colonized by clones carrying rare (traceable) plasmid variants. Patients are represented with their corresponding threeletter patient code. Circles represent clones isolated from the patient, with the fill colour indicating the bacterial species and the outline colour indicating the plasmid variant (see legend). The sequence type (ST) of each clone is indicated. Circles in the same row indicate different isolates of the same clone; the number inside the second circle indicates the number of SNPs accumulated in the complete genome relative to the first isolation.

High in vitro $p O X A-48$ conjugation rate

Despite the limitations imposed by the sensitivity and frequency of the sampling method, the four selected pOXA-48 variants with core-region SNPs demonstrated pervasive within-patient plasmid transfer. However, the specific SNPs used as genetic fingerprints might affect the conjugation ability of the plasmid, which would make it impossible to generalize the results with these variants to the most common pOXA-48 variant. To exclude this possibility, we experimentally tested the conjugation rates of the most common pOXA-48 variant and the four traceable variants by introducing the plasmids independently into the $E$. coli strain $\mathrm{J} 53$ and determining the conjugation rate of each variant in this isogenic background (Figure 6, see methods). Although conjugation rates differed slightly (ANOVA; $F=2.9, \mathrm{df}=4, P=0.037$ ), they did not differ significantly between the traceable SNP plasmid variants and the most frequent variant (Tukey multiple comparisons of means, $P>0.3$ ), indicating that all these plasmid variants have similar within-patient transfer ability. We therefore conclude that horizontal spread of pOXA-48 in the gut microbiota is the norm in colonized patients. Interestingly, and as previously reported ${ }^{28}$, the in vitro pOXA-48 conjugation rate was extremely high, with a median frequency of 0.3 transconjugants per donor after only 
350 helps to explain the frequent within-patient plasmid transfer reported here.

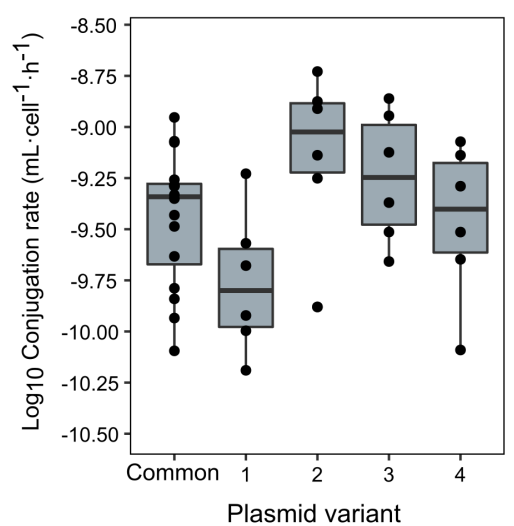

Figure 6. pOXA-48 conjugation rate. Conjugation rates of the most common pOXA-48 variant in the hospital (common, 12 biological replicates) and the four core-region SNP variants used to track within-patient plasmid transfer (6 biological replicates). Plasmid variant numbers correspond to those indicated in Figure 5. Horizontal lines inside boxes indicate median values, the upper and lower hinges correspond to the 25th and 75th percentiles, and whiskers extend to observations within 1.5 times the interquartile range.

\section{Discussion}

CPE are emerging as one of the most concerning threats to public health worldwide ${ }^{5}$. Recent studies have highlighted the central relevance of hospitals as hotspots for the dissemination of CPE among patients and for the dissemination of the carbapenemase-encoding conjugative plasmids between enterobacteria clones ${ }^{6-8}$. In this study, we performed a high-resolution epidemiological analysis to uncover the dissemination routes of the carbapenemase-encoding plasmid pOXA-48 (both at the bacterial and plasmid levels). By integrating epidemiological and genomic data, we uncovered frequent between-patient bacterial transfer and pervasive within-patient plasmid transfer. 
In light of our results, we propose that in-hospital pOXA-48 dissemination generally adheres to the following dynamics: high-risk pOXA-48-carrying enterobacteria clones, mainly K. pneumoniae ST11, spread among hospitalised patients, colonising their gut microbiota (Figures 2, 3 and 4). Once patients are colonised, the plasmid readily spreads through conjugation towards other resident members of the gut microbiota (enterobacteria such as E. coli, C. freundii, and E. cloacae, Figures 1 and 5). The plasmid's high conjugation rate increases its chances of becoming established in the gut microbiota because, even if the invading nosocomial clone is eliminated, pOXA-48 can survive in a different bacterial host. Moreover, the frequent plasmid transfer provides a test bench for new bacterium-plasmid combinations, some of which may be particularly successful associations able to persist and even disseminate towards new human hosts ${ }^{4}$. An illustrative example of these general dynamics is the case of the patient carrying plasmid variant 4 (Figure 5B; patient code TGY). This patient was first colonised by $K$. pneumoniae ST11/pOXA-48 in October 2014, and 11 days later a pOXA-48-carrying E. coli strain was isolated from the same patient (ST457). During a new admission 17 months later, a different pOXA-48-carrying E. coli (ST1722) was recovered the patient's gut microbiota. The pOXA-48 variant in all the clones carries a traceable SNP, confirming that the patient was colonized throughout the period by a pOXA-48-carrying enterobacteria, even though the plasmid had moved from its original K. pneumoniae ST11 host to E. coli clones in the gut microbiota.

Another interesting observation emerging from this study is that most of the events attributed to between-patient transmission originated from a small subset of patients (Figure 4). This result highlights the potential role of super-spreader patients in the nosocomial dissemination of $\mathrm{CPE}^{29}$. Unfortunately, given the small number of superspreader patients, we were not able to associate them to any particular epidemiological aspect, such as age or length of stay. 
An important goal of this study is to inform new and improved intervention strategies aimed at controlling the spread of carbapenem resistance in hospitals. Our results can help in the design of interventions to control OXA-48 dissemination at two levels:

(i) Between-patient. We have shown that the spread of pOXA-48-carrying enterobacteria between patients in the hospital is mainly mediated by high-risk clones commonly associated with nosocomial infections. These clones reside in hospital settings and are able to survive in the environment, creating stable reservoirs (often involving sinks ${ }^{30-32}$ ). Moreover, our results also showed that there are specific colonisation and transmission hotspots, such as individual rooms within wards (Supplementary Figure 11). We therefore propose that measures to detect and control environmental reservoirs and transfer hotspots could prevent between-patient OXA48 dissemination. Such measures could represent a useful addition to the strategies based on patient surveillance and standard precautions already applied in hospitals, and could complement and improve the outcome of contact isolation measures ${ }^{22}$.

(ii) Within-patient. A key finding of our study is the high prevalence of within-patient pOXA-48 transfer, which in turn helps to establish long-term pOXA-48 gut carriers. Preventing within-patient plasmid transfer and gut carriage is thus a particularly promising strategy for containing carbapenem resistance. This goal could be achieved either by blocking plasmid conjugation ${ }^{33}$ or, ideally, by specifically clearing pOXA-48 from the gut microbiota of patients by targeted decontamination. Decontamination strategies would aim to remove pOXA-48 plasmid or pOXA-48-carrying enterobacteria from carriers while preserving the integrity of the gut microbiota. New biotechnological advances are being made on this front. For example, CRISPR (clustered regularly interspaced short palindromic repeats) based technology can be used for targeted plasmid elimination ${ }^{34}$, and the new toxin-intein antimicrobials could be engineered to selectively remove pOXA-48-carrying clones from the microbiota ${ }^{35}$. Further work is 
urgently needed to tailor these emerging technologies into effective intervention strategies against the threat of plasmid-mediated carbapenemases.

\section{Methods}

\section{Study design and data collection}

We studied samples collected from patients admitted in a Spanish university hospital from March 4th, 2014, to March $31^{\text {st }}, 2016$, as part of an active surveillance-screening program for detecting ESBL/carbapenemase-carriers (R-GNOSIS-FP7-HEALTH-F32011-282512, www.r-gnosis.eu/) ${ }^{18,22,23,36}$. This study was approved by the Ramón y Cajal University Hospital Ethics Committee (Reference 251/13), which waived the need for informed consent from patients on the basis that the study was assessing ward-level effects and it was of minimal risk. This screening included a total of 28,089 samples from 9,275 patients admitted at 4 different wards (gastroenterology, neurosurgery, pneumology and urology) in the Ramon y Cajal University Hospital (Madrid, Spain). We used a randomly generated three-letters code for patient anonymization. Rectal samples were obtained from patients within $72 \mathrm{~h}$ of ward admission; weekly additional samples were recovered in patients hospitalised $\geq 7$ days, and a final sample at discharge was obtained in those patients with a hospital stay $\geq 3$ days (swabbing interval: gastroenterology, median 2 days, IQR 1, 6 days; neurosurgery, median 3 days, IQR 1, 7 days; pneumology, median 2 days, IQR 1, 6 days; urology, median 1 day, IQR 1, 3 days). This protocol allowed us to obtain a time sequence for each patient in the hospital.

In this paper we have focused on the subset of patients colonised by pOXA-48-carrying enterobacteria within the R-GNOSIS project. Prevalence of colonisation by OXA-48carrying enterobacteria among patients from 2014 to 2016 was $1.13 \%(105 / 9,275$ patients). pOXA-48-carrying enterobacteria were the most frequent CPE in the hospital in this period, with 171 positive isolates (Supplementary Table 1). To better 
449 characterise pOXA-48 diversity and dissemination, we included in the within-patient 450 pOXA-48 transfer analysis all the pOXA-48-carrying enterobacteria isolated from 451 patients in our hospital since it was first reported in 2012. Specifically, we included 79 additional pOXA-48 carrying enterobacteria not included in the R-GNOSIS project 453 (Supplementary Table 1).

\section{Bacterial characterisation}

455 Samples were initially characterised as previously described, following the RGNOSIS 456 protocol $^{23}$. Briefly, swabs were plated on Chromo ID-ESBL and Chrom-CARB/OXA-48 457 selective agar media (BioMérieux, France) and bacterial colonies able to grow on these media were identified by MALDI-TOF MS (Bruker Daltonics, Germany). OXA-48 production was confirmed with KPC/MBL/OXA-48 Confirm Kit test (Rosco Diagnostica, Denmark). The MicroScan automated system (Beckman Coulter, CA, USA) was used

461 for the antimicrobial susceptibility testing and the results were interpreted according to 462 EUCAST guidelines (EUCAST breakpoint v7.1, www.eucast.org). Furthermore, 463 specific bla ${ }_{0 \times A}-48$ resistance gene was identified by multiplex $\mathrm{PCR}^{37}$, and the PCR 464 products were sequenced and compared with the GenBank database.

465 Bacterial culture, DNA extraction, Illumina sequencing and PacBio sequencing

466 All pOXA-48-carrying enterobacteria isolates $(n=250)$ were grown in Lysogeny broth 467 (LB) medium at $37^{\circ} \mathrm{C}$. Genomic DNA of all the strains was isolated using the Wizard 468 genomic DNA purification kit (Promega, Madison, WI, USA), following manufacturer's 469 instructions. Whole genome sequencing was conducted at the Wellcome Trust Centre 470 for Human Genetics (Oxford, UK), using the Illumina HiSeq4000 platform with 125 471 base pair (bp) paired-end reads. Furthermore, 2 isolates and 5 specific pOXA-48 472 plasmids (K. pneumoniae isolates $\mathrm{k} 8$ and $\mathrm{k} 165$, and plasmids from $K$. pneumoniae 473 isolates k2, k164, k187, k236-1 and k273) were sequenced using the PacBio platform 474 (The Norwegian Sequencing Centre; PacBio RSII platform using P5-C3 chemistry). 
476 Trimmomatic $v 0.33^{38}$ was used to trim the Illumina sequence reads. SPAdes $v 3.9 .0^{39}$

477 was used to generate de novo assemblies from the trimmed sequence reads with the

478 -cov-cutoff flag set to 'auto'. QUAST v4.6.0 $0^{40}$ was used to generate assembly statistics.

479 All the de novo assemblies reached enough quality including total size of $5-7 \mathrm{Mb}$, the

480 total number of contigs over $1 \mathrm{~kb}$ was lower than 200 and more than $90 \%$ of the 481 assembly comprised contigs greater than $1 \mathrm{~kb}$. Prokka $\mathrm{v} 1.5^{41}$ was used to annotate 482 the de novo assemblies with predicted genes.

483

484

485

486

487

488

489

490

\section{Phylogenetic analysis and identification of STs and clustering}

Mash $\mathrm{v} 2.0^{42}$ was used to determine distances between genomes using the raw sequence reads, and a phylogeny was constructed with mashtree v0.33 ${ }^{43}$. For the analysis of the core genome (the set of homologous nucleotides present in all the isolates when mapped against the same reference) and the core sequence of the pOXA-48 plasmid, an alignment of the single nucleotide polymorphisms (SNPs) obtained with Snippy v2.5 (https://github.com/tseemann/snippy) was used to infer a phylogeny. A maximum-likelihood tree was generated using IQ-TREE with the feature of automated detection of the best evolutionary model ${ }^{44}$. All trees were visualised using the iTOL tool ${ }^{45}$. Recombination regions were identified with Gubbins ${ }^{46}$.

The seven-gene ST of all the isolates was determined using the multilocus sequence typing (MLST) tool (https://github.com/tseemann/mlst).

\section{Transmission mathematical modelling}

Our statistical model was designed based on the premises established by Crellen T, et $a l^{24}$. The objective of our model is to estimate the daily probability of acquisition of a new pOXA-48-carrying enterobacteria by a patient in the hospital. Acquisition can occur through pOXA-48-carrying bacteria acquisition (between-patient transfer), or through pOXA-48 conjugation in the gut microbiota of the patient toward a new enterobacteria host (within-patient transfer). 
We tracked all the pOXA-48-carrying enterobacteria identified in the hospital during the R-GNOSIS study period (Figure 1). This allows us to estimate and compare the acquisition of the most prevalent species, K. pneumoniae and E. coli independently. Each day in the ward, a patient can become colonised by a new pOXA-48-carrying $K$. pneumoniae or E. coli. However, as we lacked swabbing results from each day, the timing of new colonisation events with a pOXA-48-carrying clone are interval censored, and the analysis needs to account for this interval censoring ${ }^{24}$. If the probability of becoming colonised on day $\mathrm{i}$ for patient $\mathrm{j}$ is $\mathrm{p}_{\mathrm{ij}}$, the probability of remaining uncolonized is $\left(1-p_{i j}\right)$. Therefore, in interval $k$ for patient $\mathrm{j}$ consisting of $\mathrm{N}_{\mathrm{kj}}$ days, the probability of remaining uncolonized is:

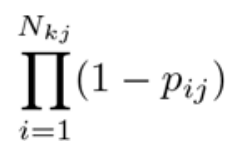

And the probability of becoming colonised $\left(v_{k j}\right)$ is the complement:

$v_{k j}=1-\prod_{i=1}^{N_{k j}}\left(1-p_{i j}\right)$

The outcome for patient $\mathrm{j}$ in interval $\mathrm{k}\left(\mathrm{X}_{\mathrm{kj}}\right)$, is either that the patient acquired a new pOXA-48-carrying enterobacteria $\left(X_{k j}=1\right)$ or did not $\left(X_{k j}=0\right)$. The likelihood is given by:

\section{$\mathrm{X}_{\mathrm{kj}} \sim \operatorname{Bernoulli}\left(\mathrm{v}_{\mathrm{kj}}\right)$}

The daily probability of becoming colonised $\left(\mathrm{p}_{\mathrm{ij}}\right)$ is related by the logit link function to a linear function of covariates $\left(\pi_{\mathrm{ij}}\right)$ :

$\Pi_{i j}=\alpha+\beta_{1} x_{1}+\beta_{2} x_{2}+\beta_{3} x_{3} \ldots$

$$
p_{\mathrm{ij}}=\exp \left(\pi_{\mathrm{ij}}\right) /\left(\exp \left(\pi_{\mathrm{ij}}\right)+1\right)
$$

Where $\mathrm{x}$ represents a vector of predictors (data) and $\beta$ is a vector of slopes (parameters) that are to be estimated. The $\beta$ coefficient can be a single parameter, or permitted to vary by ward. The range of values and the prior distributions of the different parameters are described in Supplementary Table 2. 
526 We developed and fitted models to study the probability of acquisition of pOXA-48-

527 carrying K. pneumoniae and, separately, E. coli. We included the probability of $K$.

528 pneumoniae and E. coli transferring the plasmid towards each other in the gut 529 microbiota of colonised patients. To that end, we introduced as covariates the number 530 of other patients already colonised by a pOXA-48-carrying enterobacteria each day, to 531 study the risk of between-patient transfer ( $\beta$ coefficient), and if a patient was previously 532 colonised with pOXA-48-carrying E. coli or $K$. pneumoniae, to study within patient 533 pOXA-48-transmission ( $\mathrm{Y}$ coefficient).

534 We considered five different transmission models to assess transmission of pOXA-48carrying K. pneumoniae and E. coli:

536 1) Where the daily risk of acquiring pOXA-48-carrying K. pneumoniae and E. coli 537 is constant (intercept only).

538 2) A constant term plus a between-patient transmission parameter $\beta$, where the 539 explanatory variable $\left(n_{i}\right)$ is the number of patients colonised by pOXA-48 540 enterobacteria in the four wards.

541 3) As (2) but permitting the transmission parameter $\beta$ to vary by ward $\left(\beta_{w}\right)$ and 542 considering the number of patients colonised by a pOXA-48 enterobacteria in each 543 ward $\left(n_{\text {wi }}\right)$.

544 4) As (2) but including a y parameter for the within-patient transmission, and an 545 explanatory variable $\left(\mathrm{x}_{\mathrm{i}}\right)$, which indicates if a patient had been previously colonised by 546 a pOXA-48-carrying enterobacteria from a different species (yes, $x_{i}=1 ; n o, x_{i}=0$ ).

547 5) As model (4) but permitting the transmission parameter $\beta$ to vary by ward $\left(\beta_{w}\right)$ 548 and considering the number of patients colonised by a pOXA-48 enterobacteria in each 549 ward $\left(\mathrm{n}_{\text {wi }}\right)$.

550 The probability of colonisation for individual $\mathrm{j}$ on day $\mathrm{i}$ for the respective models is 551 calculated from: 
553 Transmission Model 2: $\log i t\left(p_{i j}\right)=\alpha+\beta n_{i}$

554 Transmission Model 3: $\operatorname{logit}\left(p_{i j}\right)=\alpha+\beta_{w} n_{w i}$

555 Transmission Model 4: $\operatorname{logit}\left(p_{i j}\right)=\alpha+\beta n_{i}+\gamma x_{i}$

$556 \quad$ Transmission Model 5: $\operatorname{logit}\left(p_{i j}\right)=\alpha+\beta_{w} n_{w i}+\gamma x_{i}$

557 We fitted the statistical models using Hamiltonian Markov chain Monte Carlo in Stan

558 (version 2.17.3) within the R environment (v. 3.4.3). Prior distributions were normal 559 distributions using weakly informative priors ${ }^{24}$. Model comparison was performed with 560 widely applicable information criterion (WAIC, Supplementary Table 2). The model that 561 best fits our data is model number 5 . We use $95 \%$ credible intervals (Crls) to 562 summarise uncertainty in posterior distributions. Daily probabilities calculated with 563 model 5 are presented in Supplementary Table 3.

\section{Identification of transmission routes among patients}

565 We applied SCOTTI ${ }^{26}$, a structured coalescent-based tool for reconstructing 566 transmission, to the dominant $K$. pneumoniae and E. coli STs (with more than four isolates: K. pneumoniae ST11, ST15, ST307 and E. coli ST10), combining epidemiological and genomic data. We used the genome alignment, avoiding the recombination regions after the gubbins analysis ${ }^{46}$, as input to SCOTTI, together with the first date when an isolate was detected in a patient, and the start and end date of each patient's infection risk period (Supplementary Table 1). Due to the possibility of

572 transmission events between wards, we established a hierarchical analysis. First, we 573 applied SCOTTI to the patients/genomes included in each ward to identify 574 transmission routes within each ward, and second, we analysed the data of the 4 wards 575 combined to identify additional transmission events between wards (Supplementary 576 Figures 3-10).

\section{Identification of within-patient transmission routes of specific plasmid variants}

578 In order to confirm within-patient plasmid transfer we studied specific pOXA-48 variants 579 across the different isolates. The sequences belonging to pOXA-48 plasmid were 
mapped using the complete sequence of one of the plasmid sequenced by PacBio as reference (from K. pneumoniae k8), and the different variants and SNPs were identified using Snippy v2.5 (https://github.com/tseemann/snippy). We first analysed the degree of genetic variation in the plasmid among all the 250 bacterial clones. We compared the pOXA-48 variants sharing a core region of at least $60 \mathrm{~kb}(>90 \%$ of the whole sequence, $n=218$, Supplementary Table 1$)$. We investigated cases where a variant of the plasmid carrying a "rare" traceable SNP is present in different clones (from different species). We found four plasmid variants present in different bacterial species and, in all cases, different species carrying the same plasmid variant were isolated from the same patient (Figure 5B). For those patients, we estimated the probability of those strains being acquired by independent subsequent transmissions events, assuming a random distribution of plasmid-carrying strains across patients. Analyses were performed using R (Version 3.4.2) (www.R-project.org).

\section{Conjugation assays}

An initial conjugation round was performed to introduce pOXA-48 plasmids variants into E. coli $\mathrm{J}^{47} 3^{47}$ (a sodium azide resistant laboratory mutant of E. coli K-12). pOXA48-carrying wild type strains (donors) and E. coli J53 (recipient) were streaked from freezer stocks onto solid LB agar medium with selective pressure (ertapenem 0.5 $\mu \mathrm{g} / \mathrm{ml}$ and sodium azide $100 \mu \mathrm{g} / \mathrm{ml}$, respectively) and incubated overnight at $37^{\circ} \mathrm{C}$. Three donor colonies and one recipient colony were independently inoculated in $2 \mathrm{ml}$ of LB in $15-\mathrm{ml}$ culture tubes and incubated for $1.5 \mathrm{~h}$ at $37^{\circ} \mathrm{C}$ and $225 \mathrm{rpm}$. After growth, donor and recipient cultures were collected by centrifugation (15 $\mathrm{min}, 1500 \mathrm{~g}$ ) and cells were re-suspended in each tube with $300 \mu$ of sterile $\mathrm{NaCl} 0.9 \%$. Then, the suspensions were mixed in a 1:1 proportion, spotted onto solid LB medium and incubated at $37^{\circ} \mathrm{C}$ for 1.5 hours. Transconjugants were selected by streaking the conjugation mix on LB with ertapenem $(0.5 \mu \mathrm{g} / \mathrm{ml})$ and sodium azide $(100 \mu \mathrm{g} / \mathrm{ml})$. The transconjugants were verified by blaoxA-48 gene amplification by PCR as previously 
607 described ${ }^{11}$. For the isogenic conjugation experiments, the five different E. coli J53

608 carrying pOXA-48 plasmid variants acted as independent donors, and a

609 chloramphenicol resistant version of J53 developed in our lab was used as the

610 recipient strain. 6 colonies of each donor and recipient strains were independently

611 inoculated in $2 \mathrm{ml}$ of LB in 15-ml culture tubes and incubated overnight at $37^{\circ} \mathrm{C}$ and

$612225 \mathrm{rpm}$. Each culture was used next day to inoculate $5 \mathrm{ml}$ of LB in $50-\mathrm{ml}$ culture tubes

613 (1:100 dilution). After 1 hour of incubation at $37^{\circ} \mathrm{C}$ and 225 r.p.m, the pellets were

614 collected by centrifugation (15 min, $1500 \mathrm{~g}$ ) and cells were re-suspended in each tube 615 with $300 \mu \mathrm{l}$ of sterile $\mathrm{NaCl} 0.9 \%$. Donor and recipient suspensions were mixed in a 1:1

616 proportion and plated on a sterile nitrocellulose filter $(0.45 \mu \mathrm{m})$ on LB agar medium and

617 incubated at $37^{\circ} \mathrm{C}$ for 1 hour. Simultaneously, each culture was plated on selecting

618 agar for donors, recipient and transconjugants as controls (carbenicillin $100 \mu \mathrm{g} / \mathrm{ml}$,

619 chloramphenicol $50 \mu \mathrm{g} / \mathrm{ml}$ and a combination of both respectively). After 1 hour of

620 incubation at $37^{\circ} \mathrm{C}$, the filter contents were re-suspended in $2 \mathrm{ml}$ of sterile $\mathrm{NaCl} 0.9 \%$,

621 diluted and plated on selective agar for donors, recipient and transconjugants.

622 Transconjugants were verified by PCR as described above. Conjugation rates were

623 determined using the end-point method $^{48,49}$ (Figure 6), and the frequencies of 624 transconjugants per donor were calculated from the same data (Supplementary Figure $62513)$.

626

627

628

629

630

631

632

633

634

635

$636 \quad 4$

637

\section{References}

1 O’Neill, J. TACKLING DRUG-RESISTANT INFECTIONS GLOBALLY: FINAL REPORT AND RECOMMENDATIONS. Review on Antimicrobal Resistance (2016).

2 Vincent, J. L. et al. International study of the prevalence and outcomes of infection in intensive care units. JAMA 302, 2323-2329, doi:10.1001/jama.2009.1754 (2009).

3 Partridge, S. R., Kwong, S. M., Firth, N. \& Jensen, S. O. Mobile Genetic Elements Associated with Antimicrobial Resistance. Clin Microbiol Rev 31, doi:10.1128/CMR.00088-17 (2018). 
6406 David, S. et al. Epidemic of carbapenem-resistant Klebsiella pneumoniae in Europe is 641 driven by nosocomial spread. Nat Microbiol, doi:10.1038/s41564-019-0492-8 (2019). doi:10.1126/scitranslmed.3004129 (2012).

Conlan, S. et al. Single-molecule sequencing to track plasmid diversity of hospitalassociated carbapenemase-producing Enterobacteriaceae. Sci Transl Med 6, 254ra126, doi:10.1126/scitransImed.3009845 (2014). Dissemination of the Carbapenem Resistance Gene blaKPC. Antimicrob Agents Chemother 60, 3767-3778, doi:10.1128/AAC.00464-16 (2016).

Pitout, J. D. D., Peirano, G., Kock, M. M., Strydom, K. A. \& Matsumura, Y. The Global Ascendency of OXA-48-Type Carbapenemases. Clin Microbiol Rev 33, doi:10.1128/CMR.00102-19 (2019).

654

655

656

657

Poirel, L., Heritier, C., Tolun, V. \& Nordmann, P. Emergence of oxacillinase-mediated resistance to imipenem in Klebsiella pneumoniae. Antimicrob Agents Chemother 48, 15-22, doi:10.1128/aac.48.1.15-22.2004 (2004).

Baquero, F. \& Coque, T. M. Multilevel population genetics in antibiotic resistance. FEMS Microbiol Rev 35, 705-706, doi:10.1111/j.1574-6976.2011.00293.x (2011).

13 Poirel, L., Bonnin, R. A. \& Nordmann, P. Genetic features of the widespread plasmid coding for the carbapenemase OXA-48. Antimicrob Agents Chemother 56, 559-562, doi:10.1128/AAC.05289-11 (2012).

14 Potron, A., Poirel, L., Rondinaud, E. \& Nordmann, P. Intercontinental spread of OXA48 beta-lactamase-producing Enterobacteriaceae over a 11-year period, 2001 to 2011. Euro Surveill 18, doi:10.2807/1560-7917.es2013.18.31.20549 (2013).

15 Gijon, D. et al. Early OXA-48-Producing Enterobacterales Isolates Recovered in a Spanish Hospital Reveal a Complex Introduction Dominated by Sequence Type 11 (ST11) and ST405 Klebsiella pneumoniae Clones. mSphere 5, doi:10.1128/mSphere.00080-20 (2020).

16 Pano-Pardo, J. R. et al. Infections caused by OXA-48-producing Klebsiella pneumoniae in a tertiary hospital in Spain in the setting of a prolonged, hospital-wide outbreak. J Antimicrob Chemother 68, 89-96, doi:10.1093/jac/dks364 (2013).

17 Hidalgo, L. et al. Sequence-based epidemiology of an OXA-48 plasmid during a hospital outbreak. Antimicrob Agents Chemother, doi:10.1128/AAC.01204-19 (2019).

Hernandez-Garcia, M. et al. Intestinal co-colonization with different carbapenemaseproducing Enterobacterales isolates is not a rare event in an OXA-48 endemic area. EClinicalMedicine 15, 72-79, doi:10.1016/j.eclinm.2019.09.005 (2019).

Haverkate, M. R. et al. Within-Host and Population Transmission of blaOXA-48 in K. pneumoniae and E. coli. PLoS One 10, e0140960, doi:10.1371/journal.pone.0140960 (2015).

Guo, L. et al. Nosocomial Outbreak of OXA-48-Producing Klebsiella pneumoniae in a Chinese Hospital: Clonal Transmission of ST147 and ST383. PLoS One 11, e0160754, doi:10.1371/journal.pone.0160754 (2016). 
21 Gottig, S., Gruber, T. M., Stecher, B., Wichelhaus, T. A. \& Kempf, V. A. In vivo horizontal gene transfer of the carbapenemase OXA-48 during a nosocomial outbreak. Clin Infect Dis 60, 1808-1815, doi:10.1093/cid/civ191 (2015).

22 Maechler, F. et al. Contact isolation versus standard precautions to decrease acquisition of extended-spectrum beta-lactamase-producing Enterobacterales in noncritical care wards: a cluster-randomised crossover trial. Lancet Infect Dis, doi:10.1016/S1473-3099(19)30626-7 (2020).

23 Hernandez-Garcia, M. et al. Characterization of carbapenemase-producing Enterobacteriaceae from colonized patients in a university hospital in Madrid, Spain, during the R-GNOSIS project depicts increased clonal diversity over time with maintenance of high-risk clones. J Antimicrob Chemother 73, 3039-3043, doi:10.1093/jac/dky284 (2018).

24 Crellen, T. et al. Transmission dynamics and control of multidrug-resistant Klebsiella pneumoniae in neonates in a developing country. Elife 8, doi:10.7554/eLife.50468 (2019).

25 Holt, K. E. et al. Genomic analysis of diversity, population structure, virulence, and antimicrobial resistance in Klebsiella pneumoniae, an urgent threat to public health. Proc Natl Acad Sci U S A 112, E3574-3581, doi:10.1073/pnas.1501049112 (2015).

26 De Maio, N., Wu, C. H. \& Wilson, D. J. SCOTTI: Efficient Reconstruction of Transmission within Outbreaks with the Structured Coalescent. PLoS Comput Biol 12, e1005130, doi:10.1371/journal.pcbi.1005130 (2016).

27 Cerqueira, G. C. et al. Multi-institute analysis of carbapenem resistance reveals remarkable diversity, unexplained mechanisms, and limited clonal outbreaks. Proc Natl Acad Sci U S A 114, 1135-1140, doi:10.1073/pnas.1616248114 (2017).

28 Potron, A., Poirel, L. \& Nordmann, P. Derepressed transfer properties leading to the efficient spread of the plasmid encoding carbapenemase OXA-48. Antimicrob Agents Chemother 58, 467-471, doi:10.1128/AAC.01344-13 (2014).

29 Lloyd-Smith, J. O., Schreiber, S. J., Kopp, P. E. \& Getz, W. M. Superspreading and the effect of individual variation on disease emergence. Nature 438, 355-359, doi:10.1038/nature04153 (2005).

30 Kizny Gordon, A. E. et al. The Hospital Water Environment as a Reservoir for Carbapenem-Resistant Organisms Causing Hospital-Acquired Infections-A Systematic Review of the Literature. Clin Infect Dis 64, 1435-1444, doi:10.1093/cid/cix132 (2017).

31 Decraene, V. et al. A Large, Refractory Nosocomial Outbreak of Klebsiella pneumoniae Carbapenemase-Producing Escherichia coli Demonstrates Carbapenemase Gene Outbreaks Involving Sink Sites Require Novel Approaches to Infection Control. Antimicrob Agents Chemother 62, doi:10.1128/AAC.01689-18 (2018).

32 Mathers, A. J. et al. Intensive Care Unit Wastewater Interventions to Prevent Transmission of Multispecies Klebsiella pneumoniae Carbapenemase-Producing Organisms. Clin Infect Dis 67, 171-178, doi:10.1093/cid/ciy052 (2018).

33 Getino, M. \& de la Cruz, F. Natural and Artificial Strategies To Control the Conjugative Transmission of Plasmids. Microbiol Spectr 6 , doi:10.1128/microbiolspec.MTBP-0015-2016 (2018).

34 Bikard, D. et al. Exploiting CRISPR-Cas nucleases to produce sequence-specific antimicrobials. Nat Biotechnol 32, 1146-1150, doi:10.1038/nbt.3043 (2014). 
35 Lopez-Igual, R., Bernal-Bayard, J., Rodriguez-Paton, A., Ghigo, J. M. \& Mazel, D. Engineered toxin-intein antimicrobials can selectively target and kill antibioticresistant bacteria in mixed populations. Nat Biotechnol 37, 755-760, doi:10.1038/s41587-019-0105-3 (2019).

36 Diaz-Agero Perez, C. et al. Local prevalence of extended-spectrum beta-lactamase (ESBL) producing Enterobacteriaceae intestinal carriers at admission and coexpression of ESBL and OXA-48 carbapenemase in Klebsiella pneumoniae: a prevalence survey in a Spanish University Hospital. BMJ Open 9, e024879, doi:10.1136/bmjopen-2018-024879 (2019).

37 Ruiz-Garbajosa, P. et al. A single-day point-prevalence study of faecal carriers in long-term care hospitals in Madrid (Spain) depicts a complex clonal and polyclonal dissemination of carbapenemase-producing Enterobacteriaceae. J Antimicrob Chemother 71, 348-352, doi:10.1093/jac/dkv355 (2016).

38 Bolger, A. M., Lohse, M. \& Usadel, B. Trimmomatic: a flexible trimmer for Illumina sequence data. Bioinformatics 30, 2114-2120, doi:10.1093/bioinformatics/btu170 (2014).

39 Bankevich, A. et al. SPAdes: a new genome assembly algorithm and its applications to single-cell sequencing. J Comput Biol 19, 455-477, doi:10.1089/cmb.2012.0021 (2012).

40 Gurevich, A., Saveliev, V., Vyahhi, N. \& Tesler, G. QUAST: quality assessment tool for genome assemblies. Bioinformatics 29, 1072-1075, doi:10.1093/bioinformatics/btt086 (2013).

41 Seemann, T. Prokka: rapid prokaryotic genome annotation. Bioinformatics 30, 20682069, doi:10.1093/bioinformatics/btu153 (2014).

42 Ondov, B. D. et al. Mash: fast genome and metagenome distance estimation using MinHash. Genome Biol 17, 132, doi:10.1186/s13059-016-0997-x (2016).

43 Katz LS, G. T., Morrison SS, Caravas JA, Zhang S, Den Bakker HC, Deng X, Carleton HA. . Mashtree: a rapid comparison of whole genome sequence files. The journal of open source software 4, 1762 (2019).

44 Nguyen, L. T., Schmidt, H. A., von Haeseler, A. \& Minh, B. Q. IQ-TREE: a fast and effective stochastic algorithm for estimating maximum-likelihood phylogenies. Mol Biol Evol 32, 268-274, doi:10.1093/molbev/msu300 (2015).

45 Letunic, I. \& Bork, P. Interactive Tree Of Life v2: online annotation and display of phylogenetic trees made easy. Nucleic Acids Res 39, W475-478, doi:10.1093/nar/gkr201 (2011).

46 Croucher, N. J. et al. Rapid phylogenetic analysis of large samples of recombinant bacterial whole genome sequences using Gubbins. Nucleic Acids Res 43, e15, doi:10.1093/nar/gku1196 (2015).

47 Matsumura, Y., Peirano, G. \& Pitout, J. D. D. Complete Genome Sequence of Escherichia coli J53, an Azide-Resistant Laboratory Strain Used for Conjugation Experiments. Genome Announc 6, doi:10.1128/genomeA.00433-18 (2018).

48 Simonsen, L., Gordon, D. M., Stewart, F. M. \& Levin, B. R. Estimating the rate of plasmid transfer: an end-point method. $J$ Gen Microbiol 136, 2319-2325, doi:10.1099/00221287-136-11-2319 (1990). 174-182, doi:10.1016/j.plasmid.2012.01.008 (2012). 


\section{Acknowledgements}

777 This work was supported by the European Research Council under the European Union's Horizon 2020 research and innovation programme (ERC grant agreement no. 757440-PLASREVOLUTION) and by the Instituto de Salud Carlos III (co-funded by

780 European Development Regional Fund "a way to achieve Europe") grants PI16-00860 and CP15-00012. The R-GNOSIS project received financial support from European Commission (grant R-GNOSIS-FP7-HEALTH-F3-2011-282512). RC acknowledges financial support from European Commission (R-GNOSIS) and Plan Nacional de I+D+i2013-2016 and Instituto de Salud Carlos III, Subdirección General de Redes y Centros de Investigación Cooperativa, Ministerio de Economía, Industria y Competitividad, Spanish Network for Research in Infectious Diseases (REIPIRD16/0016/0011) co-financed by European Development Regional Fund "A way to achieve Europe" (ERDF), Operative program Intelligent Growth 2014-2020. BSC and TC acknowledge support from the UK Medical Research Council and Department for International Development [grant number MR/K006924/1], and BSC and PM acknowledge support under the framework of the JPIAMR - Joint

792 Programming Initiative on Antimicrobial Resistance. ASM is supported by a Miguel 793 Servet Fellowship (MS15-00012). JRB is a recipient of a Juan de la Cierva794 Incorporación Fellowship (IJC2018-035146-I) co-funded by Agencia Estatal de Investigación del Ministerio de Ciencia e Innovación. MH-G was supported with a contract from Instituto de Salud Carlos III, Spain (iP-FIS program, ref. IFI14/00022).

798 (funded by Wellcome Trust grant reference 203141/Z/16/Z) for the generation and initial processing of the sequencing data.

\section{Author contributions}

801 ASM, RLS and BC conceived the study. RC designed and supervised sampling and 802 collection of bacterial isolates. MHG, PRG collected the bacterial isolates and 
803 performed bacterial characterization. CDA and NLF collected the epidemiological data

804 and performed preliminary analyses. R-GNOSIS WP5 Study Group designed sampling

805 protocols and facilitated the training and capacity building for the collection of bacterial

806 isolates and preliminary analyses. JdLF, JRB and CdIV performed the experimental

807 work and analysed the results. RLS, BC, PM and TC performed the data analysis.

808 ASM and RLS wrote the initial draft of the manuscript. ASM, RLS, JdIF, JRB, BC, PM,

809 and TC contributed to the final version of the manuscript. All authors read and

810 approved the manuscript.

\section{Competing interests}

812 Authors declare no competing interests.

813 Data availability

814 The sequences generated and analysed during the current study are available in the

815 Sequence Read Archive (SRA) repository, BioProject ID: PRJNA626430,

816 http://www.ncbi.nlm.nih.gov/bioproject/626430.

817 Code availability

818 The code generated during the current study is available in GitHub,

819 http://www.github.com/leonsampedro/transmission_stan_code. 
bioRxiv preprint doi: https://doi.org/10.1101/2020.04.20.050476; this version posted April 22, 2020. The copyright holder for this preprint (which was not certified by peer review) is the author/funder, who has granted bioRxiv a license to display the preprint in perpetuity. It is made available under aCC-BY-NC-ND 4.0 International license.

\section{Supplementary tables}

821 Supplementary Table 2. Transmission models to study the daily probability of acquisition of pOXA-48-carrying enterobacteria by hospitalised patients.

\begin{tabular}{|c|c|c|c|c|c|c|}
\hline \multirow[b]{2}{*}{ Model } & \multirow[b]{2}{*}{ Parameters } & \multirow[b]{2}{*}{ Priors } & \multicolumn{2}{|l|}{ K. pneumoniae } & \multicolumn{2}{|l|}{ E. coli } \\
\hline & & & Posterior Median $(95 \% \mathrm{Crl})^{1}$ & WAIC ${ }^{2}$ & Posterior Median $(95 \% \mathrm{Crl})^{1}$ & WAIC \\
\hline 1 & a (intercept) & Normal $(0,10)$ & $-6.787(-6.9797,-6.6019)$ & 1411 & $-7.633(-7.9330,-7.3640)$ & 705 \\
\hline 2 & $\begin{array}{l}\text { a (intercept) } \\
B \text { (between-patient transfer) }\end{array}$ & $\begin{array}{l}\text { Normal }(0,10) \\
\text { Normal }(0,10)\end{array}$ & $\begin{array}{l}-6.897(-7.1103,-6.6990) \\
1.0611(0.4981,1.4857)\end{array}$ & 1402 & $\begin{array}{l}-7.716(-8.0609,-7.4121) \\
0.8333(-0.7172,1.5460)\end{array}$ & 704 \\
\hline 3 & $\begin{array}{l}\text { a (intercept) } \\
B \text { (between-patient transfer) } \\
\text { M (beta mean) } \\
\Sigma \text { (beta standard deviation) }\end{array}$ & $\begin{array}{l}\text { Normal }(0,10) \\
\text { Normal }(\mu, \sigma) \\
\text { Normal }(0,10) \\
\text { Normal }(0,5)\end{array}$ & $\begin{array}{l}-6.893(-7.1098,-6.7027) \\
\text { Varies by ward } \\
0.6697(-2.3853,2.6142) \\
1.5789(0.4050,6.3881)\end{array}$ & 1397 & $\begin{array}{l}-7.699(-8.0255,-7.4054) \\
\text { Varies by ward } \\
0.0665(-4.5453,2.3773) \\
1.4629(0.2166,7.6397)\end{array}$ & 706 \\
\hline 4 & $\begin{array}{l}\text { a (intercept) } \\
B \text { (between-patient transfer) } \\
\text { Y (within-patient transfer) }\end{array}$ & $\begin{array}{l}\text { Normal }(0,10) \\
\text { Normal }(0,10) \\
\text { Normal }(0,10)\end{array}$ & $\begin{array}{l}-6.919(-7.1521,-6.7088) \\
1.0724(0.3978,1.4903) \\
2.7390(1.2877,3.7049)\end{array}$ & 1394 & $\begin{array}{l}-7.827(-8.1749,-7.5305) \\
0.6875(-0.9421,1.4782) \\
3.034(2.0509,3.8361)\end{array}$ & 682 \\
\hline 5 & $\begin{array}{l}\text { a (intercept) } \\
\text { B (between-patient transfer) } \\
\text { M (beta mean) } \\
\Sigma \text { (beta standard deviation) } \\
\text { Y (within-patient transfer) }\end{array}$ & $\begin{array}{l}\text { Normal }(0,10) \\
\text { Normal }(\mu, \sigma) \\
\text { Normal }(0,10) \\
\text { Normal }(0,5) \\
\text { Normal }(0,10)\end{array}$ & $\begin{array}{l}-6.924(-7.1401,-6.7253) \\
\text { Varies by ward } \\
0.7198(-3.0451,2.9985) \\
1.5960(0.3937,6.7936) \\
2.7360(1.2051,3.7623)\end{array}$ & 1388 & $\begin{array}{l}-7.836(-8.1921,-7.5285) \\
\text { Varies by ward } \\
-0.2390(-5.9522,2.3138) \\
1.9315(0.3361,8.3896) \\
3.124(2.1074,3.9739)\end{array}$ & 682 \\
\hline
\end{tabular}

823

824 The table shows the parameters, prior and posterior distributions along with the WAIC

825 (model comparison measure where lower values indicate a better fit to data). See

826 methods for equations. Normal prior distributions show the mean and standard

827 deviation respectively within brackets. ${ }^{1} 95 \%$ Credible interval. ${ }^{2}$ Widely applicable

828 information criterion (WAIC). 
830 Supplementary Table 3. Risk factors and the daily probability for acquisition of pOXA-

831 48-carrying enterobacteria by hospitalised patients.

\begin{tabular}{|c|c|c|}
\hline \multirow{2}{*}{ Variable } & \multicolumn{2}{|c|}{$\begin{array}{c}\text { Daily Probability of Acquisition }{ }^{1} \\
\text { Posterior Median }\left(95 \% \mathrm{Cr}^{2}\right)\end{array}$} \\
\cline { 2 - 3 } & K. pneumoniae & E. coli \\
\hline Baseline & $0.00098(0.00079,0.00120)$ & $0.00040(0.00028,0.00054)$ \\
\hline Other patients colonised $(ß)^{3}$ & & \\
\hline Neurosurgery & $0.00650(0.00276,0.01089)$ & $0.00012(2.39 \mathrm{E}-9,0.00117)$ \\
\hline Gastroenterology & $0.00163(0.00034,0.00385)$ & $0.00072(4.86 \mathrm{E}-5,0.00254)$ \\
\hline Pneumology & $0.00268(0.00115,0.00445)$ & $0.00099(0.00012,0.00216)$ \\
\hline Urology & $0.00056(3.34 \mathrm{E}-7,0.00381)$ & $0.00016(5.88 \mathrm{E}-9,0.00178)$ \\
\hline Previously colonised patient $(\mathrm{Y})^{4}$ & $0.01489(0.00330,0.04009)$ & $0.00889(0.00336,0.01917)$ \\
\hline
\end{tabular}

832

833 Daily probability of acquisition of pOXA-48-carrying K. pneumoniae or E. coli among 834 patients admitted to the four different wards during the study period of the R-GNOSIS 835 project. ${ }^{1}$ Probability of acquisition is the posterior intercept plus the posterior 836 coefficients transformed onto the probability scale. ${ }^{2}$ Credible Interval of Posterior 837 Distribution. ${ }^{3}$ Risk of between-patient transfer: other patients already colonised by a 838 pOXA-48-carrying enterobacteria each day, divided by ward. ${ }^{4}$ Risk of within-patient 839 transfer: patient previously colonised by a pOXA-48-carrying enterobacteria of the 840 different species. 
bioRxiv preprint doi: https://doi.org/10.1101/2020.04.20.050476; this version posted April 22, 2020. The copyright holder for this preprint (which was not certified by peer review) is the author/funder, who has granted bioRxiv a license to display the preprint in perpetuity. It is made available under aCC-BY-NC-ND 4.0 International license.

\section{Supplementary figures}

842 Supplementary Figure 1. Plasmid pOXA-48.

843

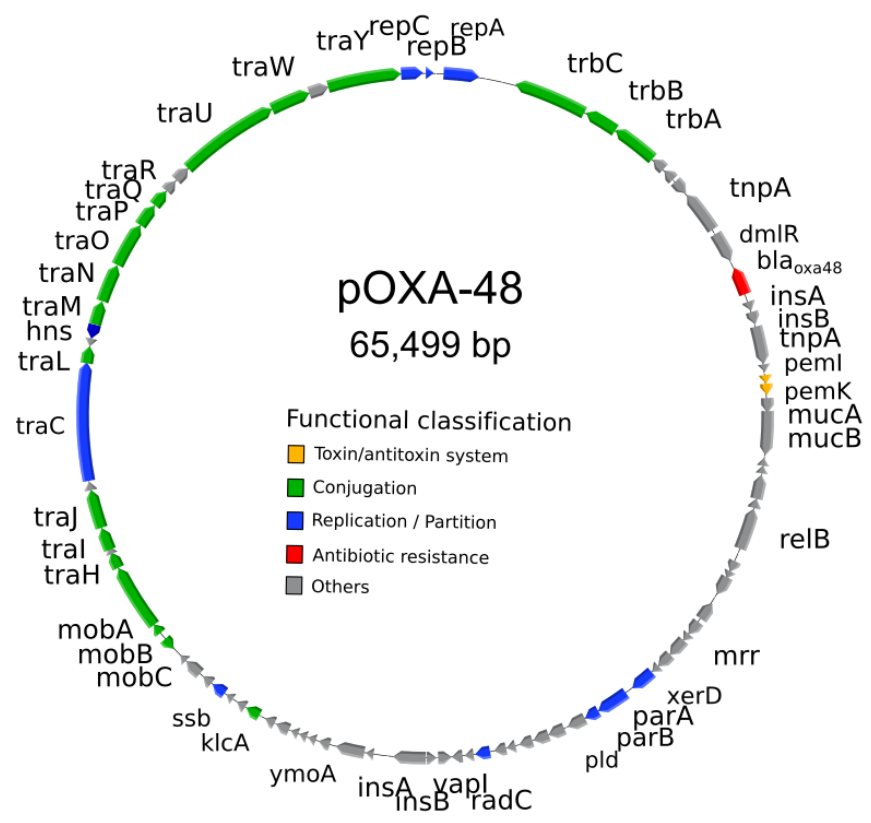

845 Schematic representation of plasmid pOXA-48. The reading frames for genes are

846 shown as arrows, with the direction of transcription indicated by the arrowhead. Arrow

847 colours indicate the functional classification of the gene (see legend). The bla oxA-48 848 gene is indicated in red. 
bioRxiv preprint doi: https://doi.org/10.1101/2020.04.20.050476; this version posted April 22, 2020. The copyright holder for this preprint

(which was not certified by peer review) is the author/funder, who has granted bioRxiv a license to display the preprint in perpetuity. It is made available under aCC-BY-NC-ND 4.0 International license.

849 Supplementary Figure 2. Phylogenetic analysis of isolates preliminary identified as $K$.

850 pneumoniae.

851

852

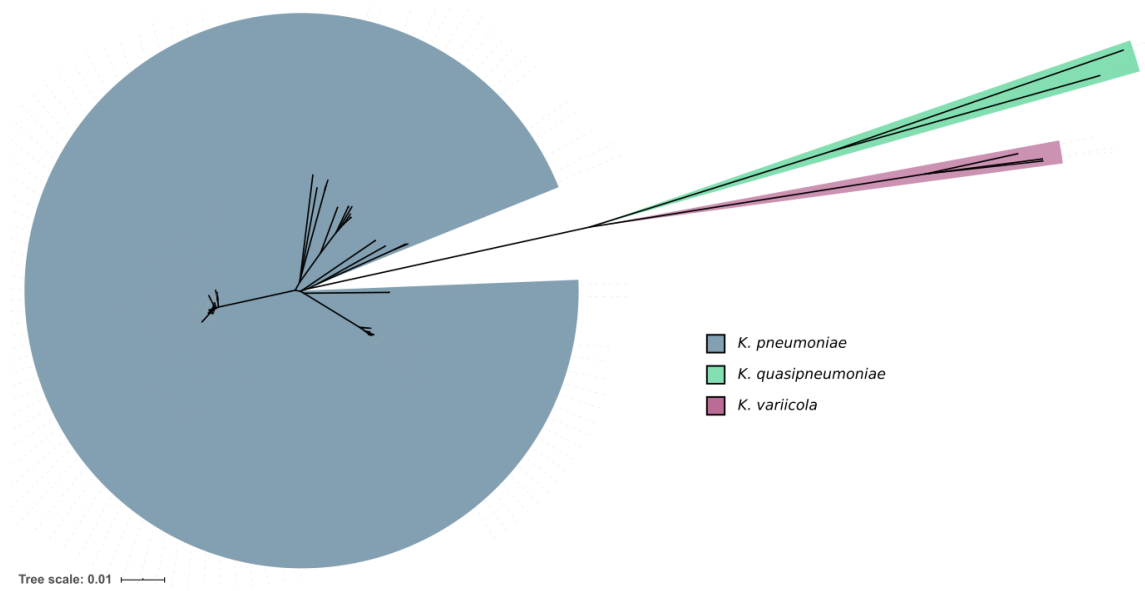

853

854 Unrooted phylogeny of 108 whole genome assemblies from the clones phenotypically

855 identified as K. pneumoniae. Branch length gives the mash distance (a measure of k-

856 mer similarity) between assemblies. Note the three distinct clusters, which are

857 considered to be separate species (distance $>0.05)$ : K. pneumoniae $(n=103), K$.

858 quasipneumoniae $(n=2)$ and $K$. variicola $(n=3)$. 
bioRxiv preprint doi: https://doi.org/10.1101/2020.04.20.050476; this version posted April 22, 2020. The copyright holder for this preprint

(which was not certified by peer review) is the author/funder, who has granted bioRxiv a license to display the preprint in perpetuity. It is made available under aCC-BY-NC-ND 4.0 International license.

859 Supplementary Figure 3. K. pneumoniae ST11 between-patient transfer dynamics in

860 the neurosurgery ward.

861

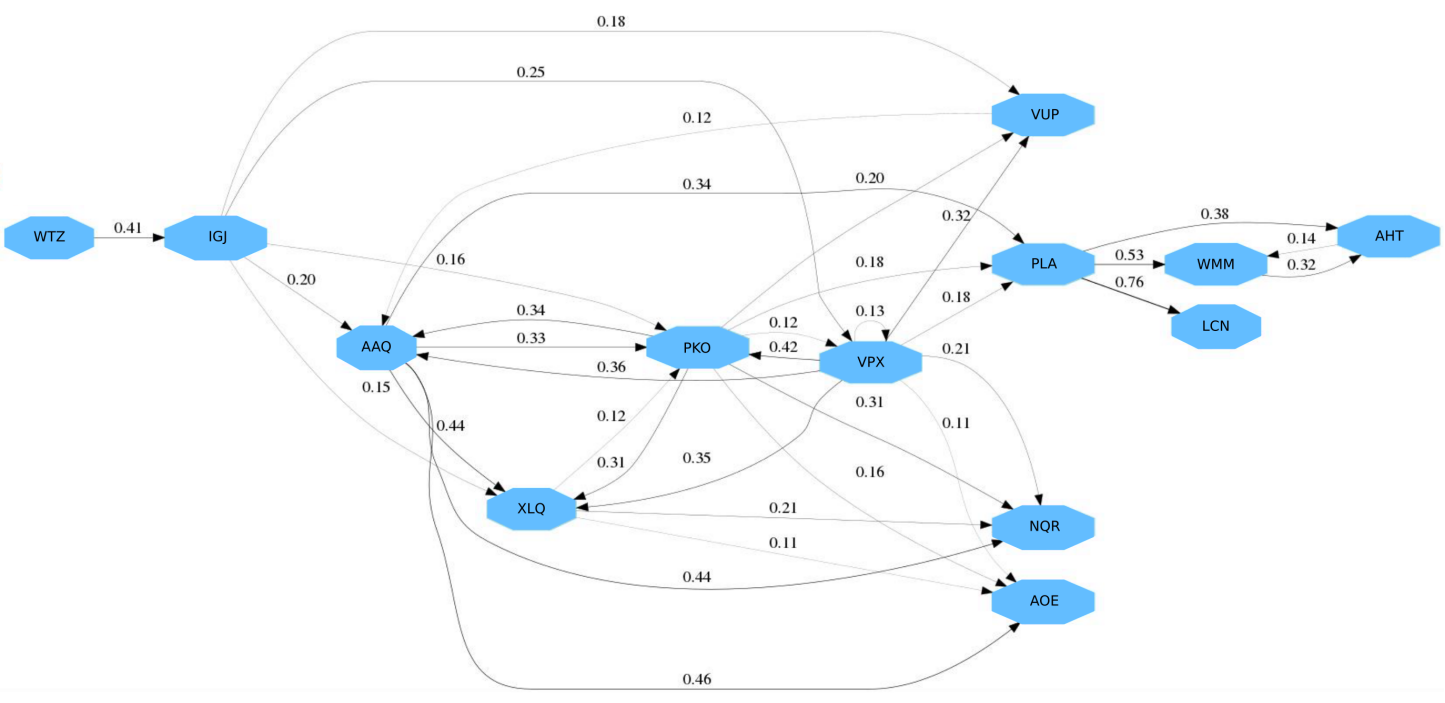

863

864 Transmission events of K. pneumoniae ST11 carrying plasmid pOXA-48 predicted by

865 SCOTTI in the neurosurgery ward. Blue boxes represent patients, with patient codes

866 indicated within the box. Lines represent the predicted between-patient transfer

867 events, and the number above the lines indicate the probability of the transfer event. 
868 Supplementary Figure 4. K. pneumoniae ST11 between-patient transfer dynamics in

869 the gastroenterology ward.

870

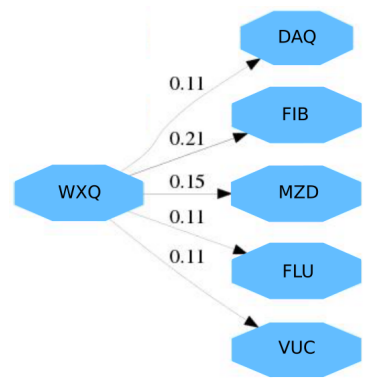

872

873 Transmission events of $K$. pneumoniae ST11 carrying plasmid pOXA-48 predicted by

874 SCOTTI in the gastroenterology ward. Blue boxes represent patients, with patient

875 codes indicated within the box. Lines represent the predicted between-patient transfer

876 events, and the number above the lines indicate the probability of the transfer event. 
877 Supplementary Figure 5. K. pneumoniae ST11 between-patient transfer dynamics in

878 the pneumology ward.

879

880

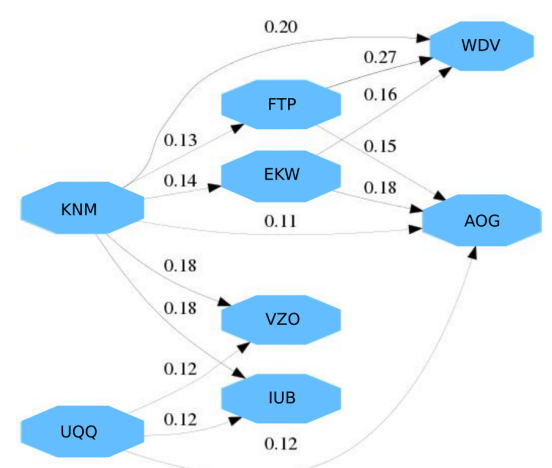

881

882 Transmission events of $K$. pneumoniae ST11 carrying plasmid pOXA-48 predicted by

883 SCOTTI in the pneumology ward. Blue boxes represent patients, with patient codes

884 indicated within the box. Lines represent the predicted between-patient transfer

885 events, and the number above the lines indicate the probability of the transfer event. 
886 Supplementary Figure 6. K. pneumoniae ST11 between-patient transfer dynamics in

887 the urology ward.

888

889

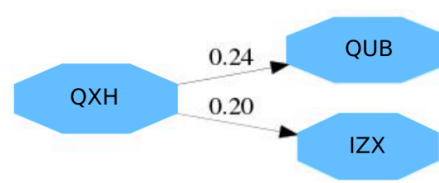

890

891

892 Transmission events of $K$. pneumoniae ST11 carrying plasmid pOXA-48 predicted by

893 SCOTTI in the urology ward. Blue boxes represent patients, with patient codes

894 indicated within the box. Lines represent the predicted between-patient transfer

895 events, and the number above the lines indicate the probability of the transfer event. 
bioRxiv preprint doi: https://doi.org/10.1101/2020.04.20.050476; this version posted April 22, 2020. The copyright holder for this preprint

(which was not certified by peer review) is the author/funder, who has granted bioRxiv a license to display the preprint in perpetuity. It is made available under aCC-BY-NC-ND 4.0 International license.

896 Supplementary Figure 7. K. pneumoniae ST11 between-patient transfer dynamics 897 across all the wards.

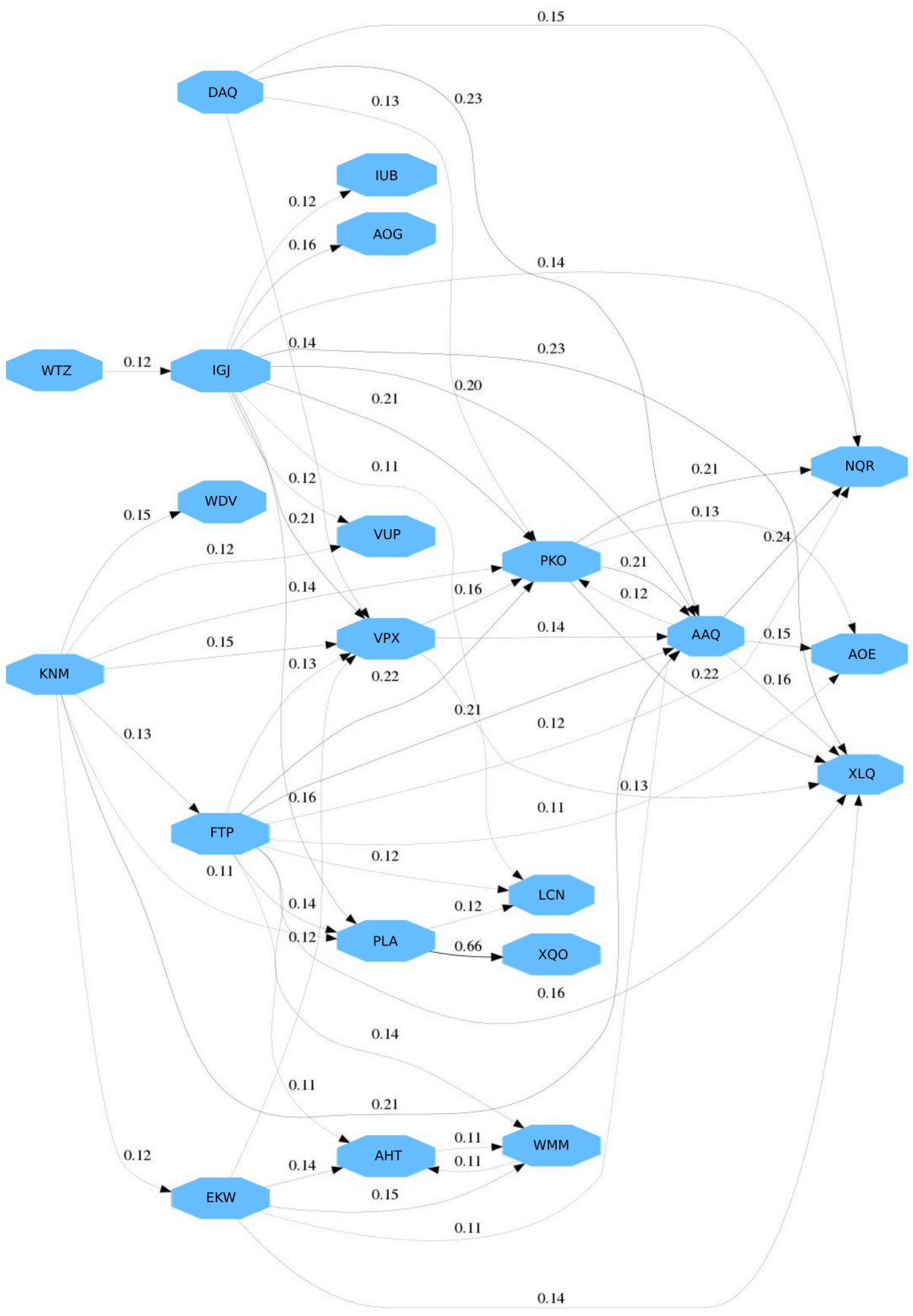

899 Transmission events of K. pneumoniae ST11 carrying plasmid pOXA-48 predicted by

900 SCOTTI when combining patients from the four wards. Blue boxes represent patients,

901 with patient codes indicated within the box. Lines represent the predicted between-

902 patient transfer events, and the number above the lines indicate the probability of the transfer event. 
904 Supplementary Figure 8. K. pneumoniae ST15 between-patient transfer dynamics

905 across all the wards.

906

907

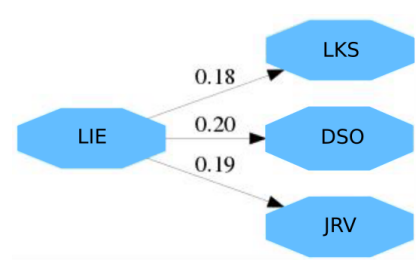

908

909 Transmission events of $K$. pneumoniae ST15 carrying plasmid pOXA-48 predicted by

910 SCOTTI when combining patients from the four wards. Blue boxes represent patients,

911 with patient codes indicated within the box. Lines represent the predicted between-

912 patient transfer events, and the number above the lines indicate the probability of the

913 transfer event. 
914 Supplementary Figure 9. K. pneumoniae ST307 between-patient transfer dynamics in

915 the pneumology ward.

916

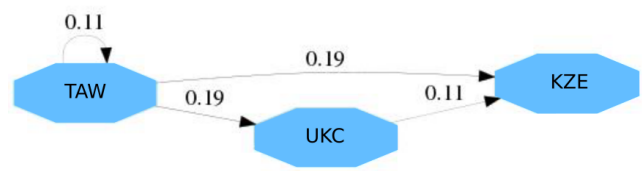

918

919 Transmission events of $K$. pneumoniae ST307 carrying plasmid pOXA-48 predicted by

920 SCOTTI in the pneumology ward. Blue boxes represent patients, with patient codes

921 indicated within the box. Lines represent the predicted between-patient transfer events, and the number above the lines indicate the probability of the transfer event. 
923 Supplementary Figure 10. E. coli ST10 between-patient transfer dynamics across all 924 the wards.

925

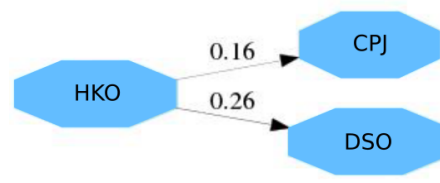

928 Transmission events of E. coli ST10 carrying plasmid pOXA-48 predicted by SCOTTI

929 when combining patients from the four wards. Blue boxes represent patients, with 930 patient codes indicated within the box. Lines represent the predicted between-patient 931 transfer events, and the number above the lines indicate the probability of the transfer event. 
933 Supplementary Figure 11. Spatiotemporal distribution of patients colonised by $K$.

934 pneumoniae ST11 in the neurosurgery ward.

935

936

937 Distribution of patients colonised by pOXA-48-carrying $K$. pneumoniae ST11 in the 938

neurosurgery ward. Each row represents a patient and the colour segments represent the length of stay in the hospital (from admission to discharge). The colours of the segments represent the different rooms within the ward (see legend). Arrows represent transmission events predicted by SCOTTI. Line thickness represents the probability of the transmission predicted by SCOTTI. The number to the right of the arrowhead indicates the number of SNPs between the complete genomes of the pair of clones involved in the putative transmission event. Note that 6 out of 16 patients shared room $\mathrm{G}$ in overlapping stays.

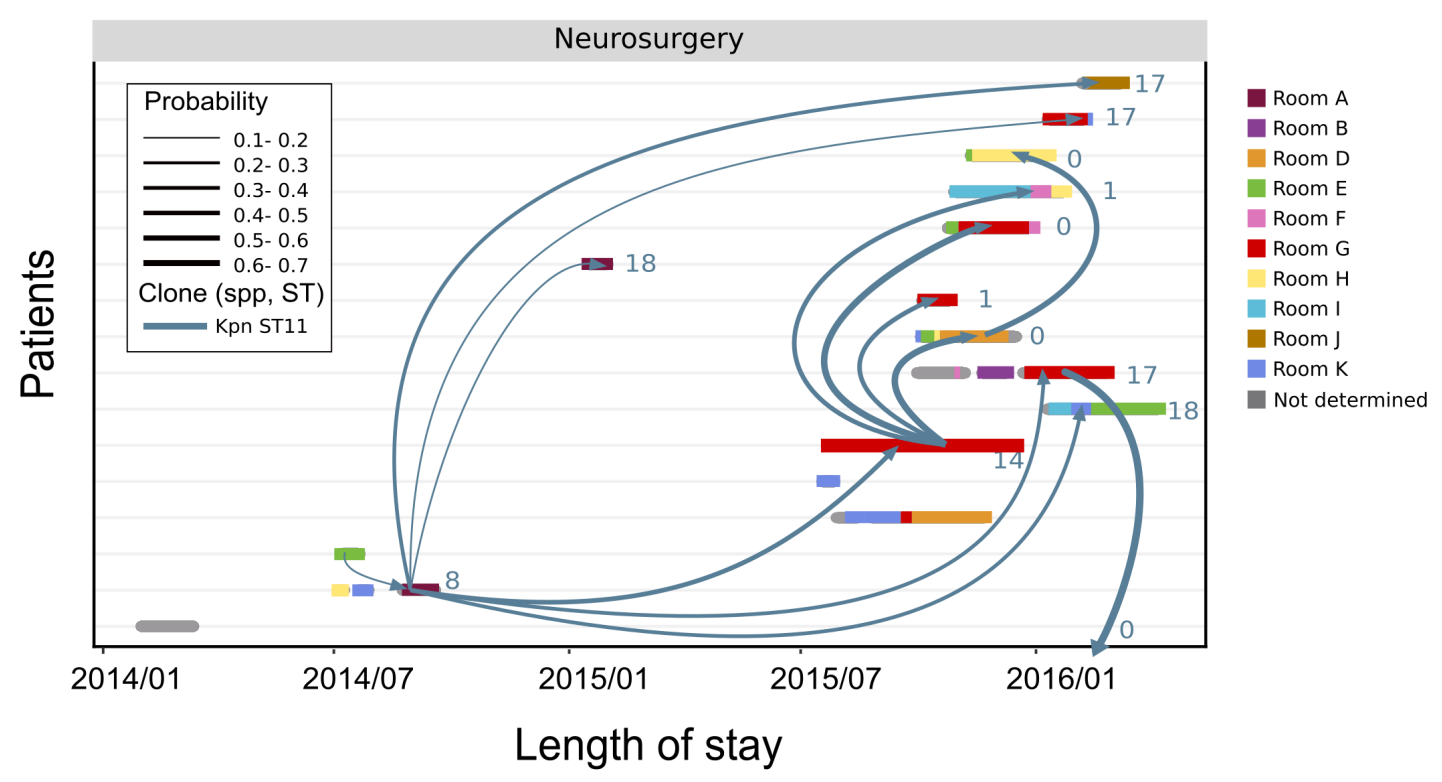


bioRxiv preprint doi: https://doi.org/10.1101/2020.04.20.050476; this version posted April 22, 2020. The copyright holder for this preprint (which was not certified by peer review) is the author/funder, who has granted bioRxiv a license to display the preprint in perpetuity. It is made available under aCC-BY-NC-ND 4.0 International license.

946 Supplementary Figure 12. pOXA-48-carrying enterobacteria analysed in this study.

947
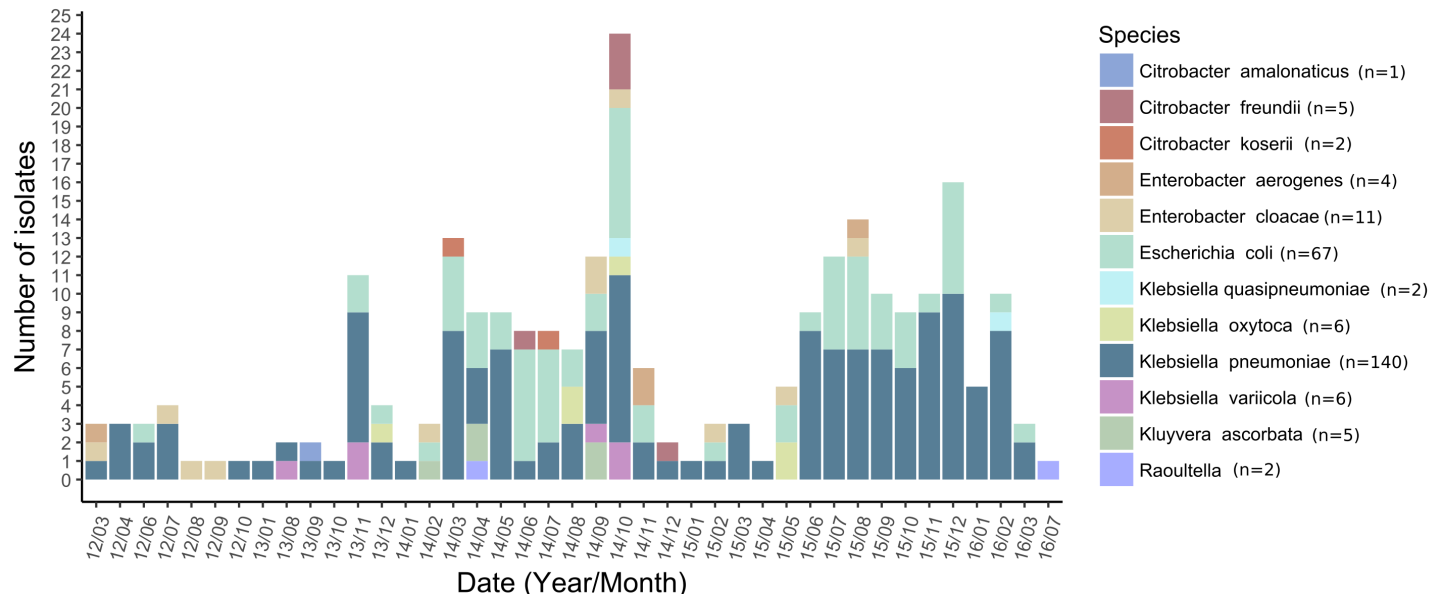

950 Representation of the 250 pOXA-48-carrying clones isolated in the hospital from the

951 first description till the end of the study period. The colour code indicates the species

952 of the pOXA-48-carrying enterobacteria as indicated in the legend. 
bioRxiv preprint doi: https://doi.org/10.1101/2020.04.20.050476; this version posted April 22, 2020. The copyright holder for this preprint

(which was not certified by peer review) is the author/funder, who has granted bioRxiv a license to display the preprint in perpetuity. It is made available under aCC-BY-NC-ND 4.0 International license.

953 Supplementary Figure 13. Frequency of conjugation of plasmid pOXA-48.

954

955

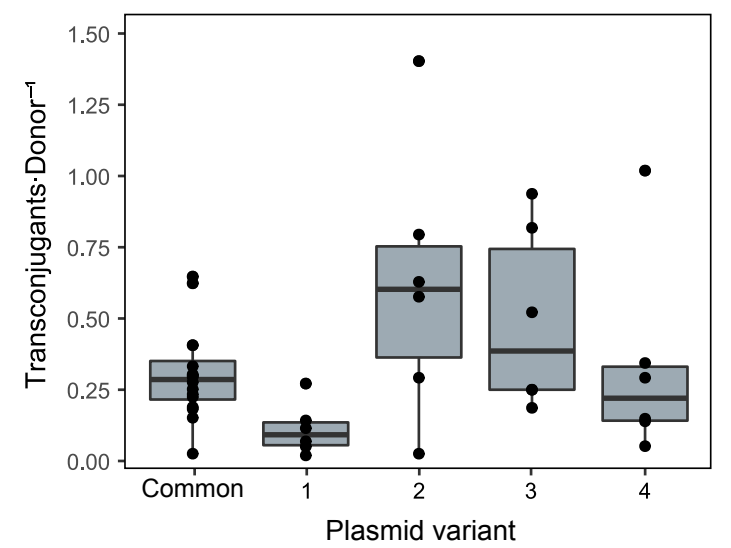

956

957 Conjugation frequencies (transconjugants per donor) of the most common pOXA-48

958 variant in the hospital (common, $n=12$ biological replicates) and the four variants with

959 SNPs in the core region used to track within-patient plasmid transfer $(n=6$ biological

960 replicates). Plasmid variant numbers correspond to those indicated in Figure 5. The

961 line inside the box marks the median. The upper and lower hinges correspond to the

962 25th and 75th percentiles and whiskers extend to observations within 1.5 times the

963 interquartile range. The data presented here is the same as in figure 6 , but represented

964 as conjugation frequency instead of rate. 